\title{
Imprimitive groups highly transitive on blocks *广
}

\author{
Claudio G. Bartolone Silvia Musumeci Karl Strambach
}

\begin{abstract}
We classify imprimitive groups acting highly transitively on blocks and satisfying conditions common in geometry. They can be realized as suitable subgroups of twisted wreath products.
\end{abstract}

In contrast to the theory of primitive permutation groups, the literature for imprimitive groups appears needy. The best known construction principle for imprimitive groups is given by wreath products of two groups $U$ and $\bar{G}$.

Suppose that $U$ is a vector space and let $V$ be the direct sum $\bigoplus_{i=1}^{t} U_{i}$ of $t$ copies of $U$. If $\bar{G}$ is a subgroup of the symmetric group $\mathbf{S}_{t}$ acting transitively on the set $\left\{U_{1}, \ldots, U_{t}\right\}$ of components of $V$ and $\alpha: \bar{G}_{U_{t}} \rightarrow \mathbf{G L}(U)$ is a linear representation of the stabilizer in $\bar{G}$ of the component $U_{t}$, then the twisted wreath product $G=U \operatorname{wr}_{\alpha} \bar{G}$ may be seen as a group of affine mappings acting transitively on a suitable system of affine subspaces of $V$ and having $t$ blocks there. The abelian group $T$ consisting of all translations is the corresponding inertial group, i.e. the normal subgroup of $G$ leaving each block fixed. Restricting to a $\bar{G}$-invariant subspace $W$ of $V$, for which there is an integer $m \leq t$ such that the projection $W \longrightarrow \bigoplus_{r=i_{1}}^{i_{m}} U_{r}$ is an isomorphism with respect to any $m$-subset of $\{1, \ldots, t\}$, we obtain a subgroup of $G$ having the same system of blocks as $G$ and inertial group given by the translations corresponding to $W$. In particular, the inertial group induces a regular action on each block and acts sharply transitively on $m$-tuples of independent points, i.e. points no two of which lying in the same block. If the representation $\alpha$ is transitive, then $U$ is finite and the stabilizer of a block is 2-transitive on it.

\footnotetext{
${ }^{*}$ Research supported by M.U.R.S.T..

†2000 Mathematics Subject Classification Primary 20B22, Secondary 20B20, 20C30, 51B05, $51 \mathrm{D} 99$.
} 
Conversely, let $G$ be an imprimitive permutation group having a finite number $t$ of blocks and assume that the stabilizer $G_{\Delta}$ in $G$ of a block $\Delta$ acts 2-transitively on it. Suppose that the inertial group $N$ induces a sharply transitive group on each block and moves uniquely any $m$-tuple of independent points onto any other one of the same type, for some $m \leq t$. Then each block $\Delta$ is a vector space of finite dimension over a prime field and $G$ can be embedded into a twisted wreath product $\Delta \operatorname{wr}_{\alpha} G / N$, where $\alpha$ is a transitive linear representation of $G_{\Delta} / N$ on $\Delta$.

In geometry imprimitive actions play an important role. For instance, the group of automorphisms of a chain geometry ([1]) is an imprimitive group operating sharply transitively on triples of independent points, such that the stabilizer of a block is 2-transitive on it. This motivated us to study imprimitive permutation groups which are highly transitive on blocks and satisfy conditions common in geometry. In particular, we classify all imprimitive permutation groups $\mathrm{G}=(G, \Omega)$ fulfilling the following conditions for some integer $m>3$ :

i) the inertial group $N$ induces a sharply transitive action on each block;

ii) given two ordered $m$-tuples $\left(X_{1}, \ldots, X_{m}\right),\left(Y_{1}, \ldots, Y_{m}\right) \in \Omega^{m}, X_{i}$ and $Y_{i}$ lying in the same block $\Delta_{i}$, there is just one element in $N$ moving $\left(X_{1}, \ldots, X_{m}\right)$ onto $\left(Y_{1}, \ldots, Y_{m}\right)$, provided $\Delta_{1}, \ldots, \Delta_{m}$ are distinct blocks;

iii) the stabilizer in $G$ of a block has a 2-transitive action on it;

$i v$ ) the group $G / N$ is finite and acts $m$-transitively on the set $\bar{\Omega}$ of blocks.

We represent these groups as subgroups of twisted wreath products. Using Kantor's, Hering's and Liebeck's results on transitive linear representations of finite simple groups ([7], [9] and [11]), we prove that the size of a block is 2,3 , 4 , or 16 and the permutation group $\bar{G}=(G / N, \bar{\Omega})$ is either the symmetric group $\mathbf{S}_{m}$, or the symmetric group $\mathbf{S}_{m+1}$ or the alternating group $\mathbf{A}_{m+2}$.

If each block contains at most 3 points then $\bar{G} \simeq \mathbf{S}_{m}$, or $\bar{G} \simeq \mathbf{S}_{m+1}$; in the latter case, if $G$ does not split over the inertial group $N$, then any block contains exactly 2 points and $m$ is odd.

If each block has 4 points then either $m=4$ and $\overline{\mathrm{G}} \simeq \mathbf{S}_{\mathbf{4}}$, or $m=5$ and $\overline{\mathrm{G}} \simeq \mathbf{S}_{\mathbf{5}}$; in both cases $G$ is a splitting extension of $N$ by $\mathbf{S}_{m}$.

If each block has 16 points, then either $m=4,5,6,7$ and $\overline{\mathbf{G}} \simeq \mathbf{A}_{m+2}$, or $m=6,7$ and $\overline{\mathbf{G}} \simeq \mathbf{S}_{m}$ or $m=5$ and $\overline{\mathbf{G}} \simeq \mathbf{S}_{\mathbf{6}}$. Moreover, the group $G$ splits always over $N$, apart from the last case where a further non-splitting extension occurs.

A tool for our classification is Blackburn's description of the extensions of a finite elementary abelian $p$-group by symmetric or alternating groups ([2]). 
1.1 Translation systems of imprimitivity. Let $G=(G, \Omega)$ be an imprimitive permutation group and let $\bar{\Omega}$ be the corresponding system of blocks. Points $\left\{X_{i}\right\}_{i \in I}$ of $\Omega$ are called independent if there is no block in $\bar{\Omega}$ containing any two of them. We shall say that $\bar{\Omega}$ is a translation system of imprimitivity of dimension $m(\leq|\bar{\Omega}|)$ if the inertial group $N$ of $\bar{\Omega}$, i.e. the normal subgroup of $G$ leaving each block of $\bar{\Omega}$ fixed, fulfills the following conditions 1.1.1 and 1.1.2:

1.1.1 The group $N$ induces on any block $\Delta \in \bar{\Omega}$ a sharply transitive group.

1.1.2 Let $\Delta_{1}, \ldots, \Delta_{m} \in \bar{\Omega}$ be distinct blocks and let $X_{i}, Y_{i} \in \Delta_{i}, i=1, \ldots, m$. Then, there is just one element $g \in N$ such that $g\left(X_{i}\right)=Y_{i}$ for all $i=1, \ldots, m$.

As a consequence of these two conditions we have:

1.1.3 Let $X_{1}, \ldots, X_{m}$ be independent points and denote by $N^{i}$ the stabilizer in $N$ of each of $X_{1}, \ldots, X_{i-1}, X_{i+1}, \ldots, X_{m}$. Then, $N=N^{1} \times \ldots \times N^{m}$.

Proof. Let $g \in N$. There is just one element $g_{1} \in N^{1}$ such that $g_{1}\left(X_{1}\right)=g\left(X_{1}\right)$, which means that $g_{1}^{-1} g$ fixes $X_{1}$. For $1 \leq j<m$, assume there is a unique element $g_{j} \in N^{j}$ such that $\left(\prod_{i=1}^{j} g_{i}\right)^{-1} g$ fixes $X_{1}, \ldots, X_{j}$. Then there is just one element $g_{j+1} \in N^{j+1}$ with $g_{j+1}\left(X_{j+1}\right)=\left(\prod_{i=1}^{j} g_{i}\right)^{-1} g\left(X_{j+1}\right)$, i.e. $\left(\prod_{i=1}^{j+1} g_{i}\right)^{-1} g$ fixes $X_{1}, \ldots, X_{j+1}$. As the stabilizer in $N$ of each of $X_{1}, \ldots, X_{m}$ is trivial, the inductive argument proves that $g$ can uniquely be written as $g_{1} \ldots g_{m}$.

1.1.4 Let the permutation group $\overline{\mathrm{G}}=(G / N, \bar{\Omega})$ be sharply $m$-transitive. Then, $G$ operates sharply transitively on the set of $m$-tuples of independent points.

Proof. Let $X_{1}, \ldots, X_{m}$ and $Y_{1}, \ldots, Y_{m}$ be $m$-tuples of independent points. Since $\bar{G}$ is $m$-transitive, there exists a coset $g N \in G / N$ such that the block containing $g\left(X_{i}\right)$ contains $Y_{i}$, as well $(i=1, \ldots, m)$. Then there exists $h \in N$ such that $h g\left(X_{i}\right)=Y_{i}$. Now, the sharply transitivity follows from the fact that the stabilizer of the points $X_{1}, \ldots, X_{m}$ is trivial.

1.1.5 Assume that $\bar{\Omega}$ is finite and the stabilizer $G_{\Delta}$ in $G$ of a block $\Delta$ acts 2 -transitively on it. Then $\mathrm{G}$ is finite.

Proof. Let $X \in \Delta$. Since $N_{X}$ leaves every point in $\Delta$ fixed, the finite group $G_{X} / N_{X} \simeq G_{\Delta} / N$ acts transitively on $\Delta \backslash\{X\}$, hence we have that $\Delta$ is finite. Thus $\Omega$ and $G$ are finite. 
2.1 Imprimitive groups and wreath products. Let $U$ be a vector space of finite dimension $n$ over a field $K, V=\bigoplus_{i=1}^{t} U_{i}$ be the direct sum of $t$ copies of $U$ and $\bar{G}$ be a transitive subgroup of the symmetric group $\mathbf{S}_{t}$ with respect to the natural action on the components $U_{i}$ of $V$. Assume we are given a homomorphism $\alpha: h \mapsto \alpha_{h}$ from the stabilizer $\bar{G}_{U_{t}}$ of $U_{t}$ into the general linear group GL $(U)$ of $U$. We can define an embedding of $\bar{G}$ into $\mathbf{G L}(V)$ as follows. Starting with a basis $e_{1}, \ldots, e_{n}$ of $U$, we have a basis

$$
\mathcal{B}=\left\{e_{11}, \ldots, e_{1 n}, \ldots \ldots, e_{t 1}, \ldots, e_{t n}\right\}
$$

of $V$, where $e_{i j}$ has $e_{j}$ as the $i$-th component and 0 as any other component. Also, fix an element $s_{i} \in \bar{G}$ with $s_{i}\left(U_{t}\right)=U_{i}$ (hence, $\mathcal{S}=\left\{s_{1}, \ldots, s_{t}\right\}$ is a set of representatives of the coset space $\left.\bar{G} / \bar{G}_{U_{t}}\right)$; in particular, choose $s_{t}=1_{\bar{G}}$. For any $g \in \bar{G}$ and $i=1, \ldots, t$, the transformation

$$
h_{g, i}:=s_{g(i)}^{-1} g s_{i}
$$

leaves the component $U_{t}$ stable. Thus putting

$$
\tilde{g}\left(e_{i j}\right):=\sum_{r=1}^{n} e_{g(i) r} a_{r} \text { if } \alpha_{h_{g, i}}\left(e_{t j}\right)=\sum_{r=1}^{n} e_{t r} a_{r},
$$

we have a linear mapping $\tilde{g} \in \mathbf{G L}(V)$. Now the identity $h_{g_{2} g_{1}, i}=h_{g_{2}, g_{1}(i)} h_{g_{1}, i}$ says that $g \mapsto \tilde{g}$ provides an embedding $\bar{G} \hookrightarrow \mathbf{G L}(V)$.

It has to be emphasized that the $(t n \times t n)$-matrix representing $\tilde{g}$ with respect to the basis $\mathcal{B}$ is obtained as follows: 1$)$ take the permutation $(t \times t)$-matrix corresponding to $g ; 2)$ replace the entry 1 at the place $(g(i), i)$ by the $(n \times n)$ matrix representing $\alpha_{h_{g, i}}$ (with respect to the basis $e_{1}, \ldots, e_{n}$ of $U$ ).

Consider now the group of affine transformations

$$
\tilde{G}:=\{x \mapsto v+\tilde{g}(x): v \in V, g \in \bar{G}\} .
$$

$\tilde{G}$ acts transitively on the set $\Omega:=\bigcup_{i=1}^{t}\left\{x+V_{i}: x \in V\right\}$, where $V_{i}=\bigoplus_{r \neq i} U_{r}$. Then the subsets $\Delta_{i}:=\left\{x+V_{i}: x \in V\right\}$ are blocks for a system of imprimitivity $\bar{\Omega}$ having the group of translations of $V$ as the inertial group. The fact that $\bigcap_{i=1}^{t} V_{i}=\mathbf{0}$ says that $\bar{\Omega}$ is a translation system of imprimitivity of dimension $t$.

2.1.1 Remark. The permutation group $\tilde{G}=(\tilde{G}, \Omega)$ is essentially the twisted wreath product $K^{n} \mathrm{wr}_{\alpha} \bar{G}$ of $U \simeq K^{n}$ by $\bar{G}$ over $\mathcal{S}$ and $\alpha$. If it is clear what linear representation we are using, we write simply $K^{n}$ wr $\bar{G}$. Changing the set of representatives the action on the set of blocks remains the same, but the action induced on a block changes according to (1) ([10, p. 86]).

2.1.2 Let $W$ be a $\bar{G}$-stable subspace of $V$. Then $\{x \mapsto w+\tilde{g}(x): w \in W, g \in \bar{G}\}$ provides a subgroup $\mathrm{H}=\left(K^{n} \operatorname{wr} \bar{G}\right)_{\mid W}$ of $K^{n} \operatorname{wr} \bar{G}$. Clearly if $W$ projects onto each 
component $U_{j}$, the subgroup $\mathrm{H}$ is transitive. Furthermore, if there is an integer $m \leq t$ such that $W$ is m-transversal with respect to the given decomposition $\bigoplus_{i=1}^{t} U_{i}$ of $V$, i.e. if the projection $W \longrightarrow \bigoplus_{r=i_{1}}^{i_{m}} U_{r}$ is an isomorphism for any $m$-subset $\left\{i_{1}, \ldots, i_{m}\right\}$ of $\{1, \ldots, t\}$ (hence $\operatorname{dim} W=m n$ ), then $\bar{\Omega}$ is a translation system of imprimitivity of dimension $m$ for $\mathrm{H}$. The stabilizer of a block is 2-transitive on it just if $\alpha$ is transitive.

2.2 Now we want discuss the converse. So let $G=(G, \Omega)$ be a transitive permutation group endowed with a finite translation system of imprimitivity $\bar{\Omega}=\left\{\Delta_{1}, \ldots, \Delta_{t}\right\}$ of dimension $m$ and assume the stabilizer $G_{\Delta_{i}}$ of a block $\Delta_{i}$ to be 2-transitive on it. According to 1.1.5, G must be finite.

Let $\left|\Delta_{i}\right|=k$. Then $|\Omega|=t k$ and we may regard $\mathrm{G}$ as a subgroup of $\mathbf{S}_{t k}$; more precisely, $G$ is contained in the subgroup $F$ preserving $\bar{\Omega}$. Let $M$ and $N$ be the inertial groups in $F$ and $G$, respectively, corresponding to $\bar{\Omega}$; of course $(M, \Omega)$ is isomorphic to the direct product of $t$ copies of $\mathbf{S}_{k}$. Let $X_{i} \in \Delta_{i}$; then there is in $M$ a subgroup $U_{i}$ acting on $\Delta_{i}$ as $N / N_{X_{i}}$ and leaving any other block point-wise fixed. Clearly $N$ is contained in the direct product $V=U_{1} \times \ldots \times U_{t}$.

Since $U_{i}$ acts sharply transitively on $\Delta_{i}$, we can identify $\Delta_{i}$ with the factor group $V / V_{i}\left(\simeq U_{i}\right)$, where $V_{i}$ denotes the product of all $U_{r}$ with $r \neq i$. As $G_{X_{i}} / N_{X_{i}}$ normalizes $N / N_{X_{i}}$, we have that $U_{i}$ is normalized by $G_{X_{i}}$, so the transitive action of $G_{X_{i}}$ on $\Delta_{i} \backslash\left\{X_{i}\right\}$ yields a transitive group of automorphisms $\left(\simeq G_{X_{i}} / N_{X_{i}} \simeq\right.$ $\left.G_{\Delta_{i}} / N\right)$ of the finite group $V / V_{i}$. Consequently $V / V_{i} \simeq U_{i}$ has to be a vector space $G F(p)^{n}$ over the prime field $G F(p)$ for some prime $p$ and integer $n$. Furthermore, there is a transitive linear representation $\alpha: G_{\Delta_{t}} / N \longrightarrow \mathbf{G L}\left(U_{t}\right) \simeq \mathbf{G L}(n, p)$ and the twisted wreath product $\tilde{\mathrm{G}}:=G F(p)^{n} \mathrm{wr}_{\alpha} G / N$, with $V$ the associated vector space, can be considered. The group $N$ is then the subgroup of translations of $\tilde{\mathrm{G}}$ corresponding to a subspace $W$ which is $m$-transversal with respect to the decomposition $V=\bigoplus_{i=1}^{t} U_{i}$. Then $G / N$ yields a subgroup of $\mathbf{G L}(V)$ normalizing $N$ and, as an extension of a group of translations of $V$ by $G / N$, the group $G$ can be viewed as a subgroup of affinities of $\tilde{G}$. Thus we have

2.2.1 Theorem. Let $\mathrm{G}=(G, \Omega)$ be a transitive permutation group endowed with a finite translation system of imprimitivity $\bar{\Omega}=\left\{\Delta_{1}, \ldots, \Delta_{t}\right\}$ of dimension $m \leq t$ and assume that the stabilizer $G_{\Delta_{i}}$ of a block $\Delta_{i}$ acts 2-transitively on $\Delta_{i}$.

Then $\mathrm{G}$ is finite and the inertial group $N$ of $\bar{\Omega}$ is an elementary abelian $p$ group for some prime $p$. Thus there exist an integer $n>0$, a transitive linear representation $\alpha: G_{\Delta_{t}} / N \longrightarrow \mathbf{G L}(n, p)$ and an embedding

$$
\iota: \mathrm{G} \hookrightarrow \tilde{\mathrm{G}}=G F(p)^{n} \operatorname{wr}_{\alpha} G / N
$$

of permutation groups such that $\iota(N)$ is a normal subgroup of translations of $\tilde{G}$ corresponding to a subspace $W$ of dimension mn of the vector space $V$ associated 
with $\tilde{\mathrm{G}}$. In particular, there exists a decomposition of $V$ with respect to which $W$ is $m$-transversal. Also $G$ splits over $N$ just if $\iota(\mathrm{G})=\left(G F(p)^{n} \mathrm{wr}_{\alpha} G / N\right)_{\mid W}$.

2.3 If we are given an integer $t>1$, a field $K$ and a linear representation $\alpha: \mathbf{S}_{t-1} \rightarrow \mathbf{G L}\left(K^{n}\right)$ of the symmetric group $\mathbf{S}_{t-1}$, the twisted wreath product $K^{n} \operatorname{wr}_{\alpha} \mathbf{S}_{t}$ is the semidirect product of its translation group $V \simeq K^{n t}$ by a complement isomorphic to $\mathbf{S}_{t}$ of linear mappings in $\mathbf{G L}\left(K^{n t}\right)$. The easiest way to represent this complement is to take all the powers of the cycle $\sigma=(1, \ldots, t)$ as a set of representatives of the coset space $\mathbf{S}_{t} / \mathbf{S}_{t-1}$. Then the linear mapping corresponding to $\sigma$ is represented by the block matrix $I=\left(I_{i j}\right)$, where

$$
I_{i j}= \begin{cases}\mathbf{I}_{n} & \text { if } i=\sigma(j), \\ \mathbf{0}_{n} & \text { otherwise }\end{cases}
$$

where $\mathbf{I}_{n}$ and $\mathbf{0}_{n}$ are the identity and the null matrix of order $n$, respectively. Moreover, for $k=1, \ldots, t-1$, the transposition $(k k+1)$ corresponds to the block matrix $J_{k}=\left(J_{i j}^{k}\right)$ having the non-zero blocks defined by

$$
J_{i i}^{k}= \begin{cases}j_{k-i} & \text { if } i<k, \\ j_{t+k-i} & \text { if } i>k+1, \quad J_{k k+1}^{k}=j, \quad J_{k+1 k}^{k}=j^{-1},\end{cases}
$$

where $j=\alpha(12 \ldots t-2 t-1)$ and $j_{r}=\alpha(r r+1)(r=1, \ldots, t-2)$.

2.3.1 Example. In $\mathbf{G L}(4,2) \simeq \mathbf{A}_{\mathbf{8}}$ all subgroups isomorphic to $\mathbf{S}_{\mathbf{6}}$ are conjugate $([4$, p. 22]) to the symplectic group $\mathbf{S p}(4,2)$ ([8, p. 227]). Furthermore in the latter group there is, up to conjugation, just one subgroup isomorphic to $\mathbf{S}_{\mathbf{5}}$ acting transitively on $G F(2)^{4}$ ([8, p. 176]). More precisely this action is equivalent to the one of $\mathbf{P} \mathbf{L L}(2,4)$ on $G F(2)^{4}$ ([4, p. 2]). So the twisted wreath product

$$
\mathrm{G}_{6}:=G F(2)^{4} \text { wr } \mathbf{S}_{6}
$$

is uniquely determined (up to isomorphisms), if we require double transitive action on a block. We shall give a representation over $G F(4)$ of $\mathrm{G}_{6}$.

Write the elements in $G F(4)$ as $0,1, \mathbf{i}, \mathbf{i}^{2}$. Then the semilinear mappings

$$
\begin{array}{ll}
j_{1}:(x, y) \mapsto\left(x^{2}, y^{2}\right), & j_{2}:(x, y) \mapsto\left(\mathbf{i}^{2} x^{2}, \mathbf{i} y^{2}\right), \\
j_{3}:(x, y) \mapsto\left(x^{2}+y^{2}, y^{2}\right), & j_{4}:(x, y) \mapsto\left(y^{2}, x^{2}\right)
\end{array}
$$

generate $\mathbf{P} \boldsymbol{\Gamma L}(2,4)$ as they satisfy the conditions

$$
j_{k}^{2}=\left(j_{u+1} j_{u}\right)^{3}=\left(j_{u} j_{v}\right)^{2}=i d_{G F(4)^{2}} \quad(k=1,2,3,4, u=1,2,3, u-v=2,3)
$$

$\left(\left[5\right.\right.$, p. 287]). Thus, for $t=6$, we may take them as the entries $j_{r}$ in (3). Then

$$
j=j_{1} j_{2} j_{3} j_{4}:(x, y) \mapsto\left(\mathbf{i} x+\mathbf{i} y, \mathbf{i}^{2} x\right) .
$$


2.4 If we are given a twisted wreath product $K^{n} \operatorname{wr}_{\alpha} \mathbf{S}_{t}$, then a twisted wreath product $K^{n}{ }^{n} r_{\alpha} \mathbf{A}_{t}$ is also defined by restriction of $\alpha$ to $\mathbf{A}_{t-1}$. As a set of representatives of the coset space $\mathbf{A}_{t} / \mathbf{A}_{t-1}$ is as well a set of representatives of $\mathbf{S}_{t} / \mathbf{S}_{t-1}$, the group $K^{n}{ }^{\mathrm{wr}} \mathrm{r}_{\alpha} \mathbf{A}_{t}$ embeds into $K^{n} \mathrm{wr}_{\alpha} \mathbf{S}_{t}$. Thus, in view of Remark 2.1.1, replacing the set of representatives by the powers of the cycle $(1, \ldots, t)$, we obtain a subgroup of $K^{n} \mathrm{wr}_{\alpha} \mathbf{S}_{t}$ isomorphic to $K^{n} \mathrm{wr}_{\alpha} \mathbf{A}_{t}$. This subgroup is the semidirect product of the translation subgroup by the group generated by the linear mappings corresponding to the 3 -cycles $(l-1 l l+1), l=2, \ldots, t-1$, which are given by the block matrices $Q_{l}=\left(Q_{i j}^{l}\right)$ having the non-zero blocks defined by

$$
Q_{i i}^{l}=\left\{\begin{array}{ll}
q_{l-i} & \text { if } i<l-1, \\
q_{t+l-i} & \text { if } i>l+1,
\end{array} \quad Q_{l-1 l+1}^{l}=q^{\prime} q^{\prime \prime}, \quad Q_{l-1}^{l}=q^{\prime-1}, \quad Q_{l+1 l}^{l}=q^{\prime \prime-1},\right.
$$

where $q^{\prime}=\alpha(23 \ldots t-2 t-1), q^{\prime \prime}=\alpha(12 \ldots t-3 t-2)$ and $q_{s}=\alpha(s-1 s s+1)$ $(s=2, \ldots, t-2)$.

\subsubsection{Example. Consider the group}

$$
\mathrm{H}_{6}:=G F(2)^{4} \text { wr } \mathbf{A}_{6}
$$

as a subgroup of $\mathrm{G}_{6}$. In such a case we have

$$
q^{\prime}=j_{2} j_{3} j_{4}, \quad q^{\prime \prime}=j_{1} j_{2} j_{3}, \quad q_{s}=j_{s-1} j_{s} \quad(s=2,3,4) .
$$

$\mathrm{H}_{6}$ is uniquely determined as a twisted wreath product when we ask 2-transitivity on a block. This follows from $\mathbf{A}_{\mathbf{5}} \simeq \mathbf{S L}(2,4)([4, \mathrm{p} .2])$, from $[8$, p. 176] and the fact that in $\mathbf{A}_{\mathbf{8}}$ all subgroups isomorphic to $\mathbf{A}_{\mathbf{6}}$ are conjugate ([7, p. 444]).

2.5 The alternating group $\mathbf{A}_{r+2}$ is generated by elements $p_{1}, \ldots, p_{r}$ such that

$$
\begin{aligned}
& p_{1}^{3}=1, \quad p_{i}^{2}=1 \quad(i>1), \\
& \left(p_{i+1} p_{i}\right)^{3}=1 \quad(i<r), \quad\left(p_{j} p_{i}\right)^{2}=1 \quad(|i-j|>1)
\end{aligned}
$$

([5, p. 289]). Up to isomorphisms, there is just one twisted wreath product

$$
\mathrm{H}_{9}:=G F(2)^{4} \text { wr } \mathbf{A}_{\mathbf{9}}
$$

with 2-transitive action on a block. This group arises using the transitive linear representation of the stabilizer $\mathbf{A}_{\mathbf{8}}$ as $\mathbf{G L}(4,2)$. Then as the generators (8) for $\mathbf{A}_{\mathbf{8}}$ we can take the semilinear mappings of the plane $G F(4)^{2}$

$$
\begin{array}{ll}
p_{1}:(x, y) \mapsto\left(\mathbf{i} x+x^{2}+\mathbf{i}^{2} y^{2}, \mathbf{i}^{2} x^{2}+\mathbf{i} y+\mathbf{i} y^{2}\right), & p_{2}:(x, y) \mapsto\left(x+\mathbf{i}^{2} x^{2}+\mathbf{i} y^{2}, \mathbf{i} x^{2}+y+y^{2}\right), \\
p_{3}:(x, y) \mapsto\left(\mathbf{i}^{2} x+x^{2}+\mathbf{i} y+y^{2}, \mathbf{i}^{2} x^{2}+\mathbf{i} y+\mathbf{i} y^{2}\right), & p_{4}:(x, y) \mapsto\left(x+\mathbf{i} y+\mathbf{i} y^{2}, \mathbf{i}^{2} x+\mathbf{i} x^{2}+y\right), \\
p_{5}:(x, y) \mapsto\left(\mathbf{i} x+\mathbf{i} x^{2}+\mathbf{i}^{2} y+\mathbf{i} y^{2}, x+\mathbf{i} y+\mathbf{i} y^{2}\right), & p_{6}:(x, y) \mapsto\left(x+\mathbf{i}^{2} x^{2}+\mathbf{i} y, \mathbf{i}^{2} x+y+y^{2}\right) .
\end{array}
$$

In particular we may identify $p_{1}$ with $\alpha(123)$ and $p_{i}$ with $\alpha(12) \alpha(i+1 i+2)$ for $i>1$. Since the cycle $(1 \ldots 9)$ is in $\mathbf{A}_{\mathbf{9}}$, we can use it to represent $\mathbf{H}_{9}$. Thus the matrices (6) with entries 


$$
\begin{array}{llll}
q_{2}=p_{1}, & q_{3}=p_{1}^{2} p_{2}, & q_{4}=p_{2} p_{3}, & q_{5}=p_{3} p_{4}, \\
q_{6}=p_{4} p_{5}, & q_{7}=p_{5} p_{6}, & q^{\prime}=p_{1}^{2} p_{2} p_{3} p_{4} p_{5} p_{6}, & q^{\prime \prime}=p_{1} p_{2} p_{3} p_{4} p_{5}
\end{array}
$$

generate a complement of the translation group of $\mathrm{H}_{9}$. Up to isomorphisms, the alternating group $\mathbf{A}_{\mathbf{6}}$ (resp. $\mathbf{A}_{\mathbf{7}}$ ) has just one transitive linear representation on the vector space of dimension 4 over $G F(2)$, arising as the restriction to $\mathbf{A}_{\mathbf{6}}$ (resp. $\mathbf{A}_{\mathbf{7}}$ ) of the above representation of $\mathbf{A}_{\mathbf{8}}$ as the general linear group $\mathbf{G L}(4,2)$ ([7, p. 444]). Thus there are unique twisted wreath products

$$
\mathrm{H}_{7}:=G F(2)^{4} \text { wr } \mathbf{A}_{\mathbf{7}}, \quad \mathrm{H}_{8}:=G F(2)^{4} \text { wr } \mathbf{A}_{\mathbf{8}}
$$

acting 2-transitively on a block.

For $s<t$, a set of representatives of the coset space $\mathbf{A}_{s} / \mathbf{A}_{s-1}$ can be completed to a set of representatives of $\mathbf{A}_{t} / \mathbf{A}_{t-1}$. Thus, if we are given a twisted wreath product $K^{n}{ }_{{ }^{\prime} r_{\alpha}} \mathbf{A}_{t}$, the twisted wreath product $K^{n}{ } \mathrm{wr}_{\alpha} \mathbf{A}_{s}$, defined by restricting $\alpha$, can be regarded as the stabilizer of $t-s$ blocks in $K^{n} \mathrm{wr}_{\alpha} \mathbf{A}_{t}$. Hence, there are embeddings $\mathrm{H}_{7} \hookrightarrow \mathrm{H}_{8} \hookrightarrow \mathrm{H}_{9}$ which allow one to represent $\mathrm{H}_{8}$ and $\mathrm{H}_{7}$ as the stabilizers in $\mathrm{H}_{9}$ of one and two blocks, respectively.

3.1 Invariant transversal subspaces. Looking at Theorem 2.2.1, we see that transversal subspaces (in the sense of 2.1.2) play an important role in representing a permutation group $\mathrm{G}=(G, \Omega)$ with a finite translation system of imprimitivity $\bar{\Omega}=\left\{\Delta_{1}, \ldots, \Delta_{t}\right\}$ within the twisted wreath product $\tilde{\mathrm{G}}=G F(p)^{n} \operatorname{wr}_{\alpha} G / N$. The inertial group $N$ of $\bar{\Omega}$ appears as a group of translations of the vector space associated to $\tilde{G}$, more precisely as the group of translations defined through the vectors in a $m$-transversal subspace $W$ of $V$. It is straightforward that

$$
W=\left\{\left(x_{1}, \ldots, x_{m}, \sum_{j=1}^{m} f_{m+1, j}\left(x_{j}\right), \ldots, \sum_{j=1}^{m} f_{t, j}\left(x_{j}\right)\right): x_{j} \in G F(p)^{n}\right\},
$$

for suitable linear mappings $f_{i, j} \in \mathbf{G L}(n, p)$.

3.2 Let $\tilde{\mathrm{G}}=G F(3) \mathrm{wr}_{\alpha} \mathbf{S}_{m+1}$ with $\alpha$ the non-trivial representation of $\mathbf{S}_{m}$ on the 1-dimensional $G F(3)$-vector space. As in 2.3, take the powers of the cycle $\sigma=(1 \ldots m+1)$ to represent a complement isomorphic to $\mathbf{S}_{m+1}$. Then the matrix corresponding to $\sigma$ is the matrix $I$ given by (2), whereas the transposition $(1 m+1)$ is represented by the matrix $J=\left(J_{i j}\right)$, the non-zero entries of which are

$$
J_{i i}=-1 \quad \text { if } \quad 2 \leq i \leq m, \quad J_{1 m+1}=J_{m+11}=\varepsilon=(-1)^{m+1} .
$$

$I$ and $J$ generate a complement isomorphic to $\mathbf{S}_{m+1}$ of the translation group in $\tilde{G}$. Now we ask whether in $V$ there is a $\mathbf{S}_{m+1}$-invariant $m$-transversal subspace, i.e. a subspace $W$ as in 3.1, with $t=m+1, p=3, n=1$, which is invariant under 
the action of $I$ and $J$. Set $f_{i}=f_{m+1, i}$, then the condition $I(W)=W$ yields $\sum_{i=1}^{m} f_{1} f_{i}\left(x_{i}\right)+\sum_{r=2}^{m} f_{r}\left(x_{r-1}\right)=x_{m}$, i.e. $f_{1} f_{m}=i d_{G F(3)}$ and $f_{r}=(-1)^{r+1} f_{1}^{r}$. Hence $(-1)^{m+1} f_{1}^{m+1}=i d_{G F(3)}$. Since $\mathbf{G L}(1,3)$ has order 2 , the latter equation says

$$
f_{1}= \begin{cases}-i d_{G F(3)} & \text { if } m \text { is even, } \\ \pm i d_{G F(3)} & \text { if } m \text { is odd. }\end{cases}
$$

Let $f_{1}=-i d_{G F(3)}$ with $m$ odd. Then from $f_{r}=(-1)^{r+1} f_{1}^{r}$ it follows $f_{r}=-i d_{G F(3)}$ for all $r$, but in such a case $J(W) \neq W$. So we have

3.2.1 Lemma. Let $\alpha$ be the non-trivial representation of $\mathbf{S}_{m}$ on the vector line over $G F(3)$. Then, in the vector space associated with the twisted wreath product $G F(3) \mathrm{wr}_{\alpha} \mathbf{S}_{m+1}$, the hyperplane $\sum_{k=1}^{m+1}(-1)^{m(k+1)} x_{k}=0$ is the only subspace which is both $m$-transversal and stable under $\mathbf{S}_{m+1}$.

3.3 Let $\tilde{\mathrm{G}}=G F(2)^{n} \operatorname{wr}_{\alpha} \mathbf{S}_{m+1}$ and let $W$ be a $m$-transversal subspace of $V$ as in 3.1 with $t=m+1$ and $p=2$. Then $W$ is stable under the action of $\mathbf{S}_{m+1}$ just if it is stable under the transformations $I$ and $J_{k}$ defined by (2) and (3). In particular $I(W)=W$ gives $f_{i}=f_{1}^{i}(i=1, \ldots, m)$ and

$$
f_{1}^{m+1}=i d_{G F(2)^{n}},
$$

whereas $J_{k}(W)=W$ gives $j_{k}=f_{1}^{k} j f_{1}^{m-k}(k=1, \ldots, m-1)$. So

$$
j_{l-1}=f_{1}^{-1} j_{l} f_{1} \quad(l=2, \ldots, m-1) .
$$

- Let $m=4$. The symmetric group $\mathbf{S}_{4}$ has a non-faithful natural representation as $\mathbf{G L}(2,2)$. As in $\mathbf{G L}(2,2) \simeq \mathbf{S}_{\mathbf{3}}$ there is no element of order 5 , in such a case (10) says $f_{1}=i d_{G F(2)^{2}}$. Consequently (11) gives $j_{l-1}=j_{l}$, i.e. $i d_{G F(2)^{2}}=j_{l-1} j_{l}=$ $\alpha(l-1 \quad l \quad l+1)$, but there are no elements of order 3 in $\operatorname{ker} \alpha \simeq \mathbb{Z}_{2} \oplus \mathbb{Z}_{2}$.

- Let $m=5$. We have a faithful representation $\alpha: \mathbf{S}_{\mathbf{5}} \longrightarrow \mathbf{P} \mathbf{\Gamma L}(2,4)$ of $\mathbf{S}_{\mathbf{5}}$ as the collineation group of the plane over $G F(4)$. Then (10) and (11) say

$$
f_{1}=f:(x, y) \longmapsto\left(\mathbf{i} x+\mathbf{i} y+\mathbf{i} y^{2}, \mathbf{i}^{2} x+\mathbf{i}^{2} y^{2}\right),
$$

if we regard $V$ as a vector space over $G F(4)$. Thus we may identify $W$ with the $G F(4)$-subspace $\sum_{i=1}^{6} f^{i}\left(x_{i}, y_{i}\right)=0$ of $V$.

- Let $m=6$. There is a faithful representation $\alpha: \mathbf{S}_{\mathbf{6}} \longrightarrow \mathbf{S p}(4,2)$ of $\mathbf{S}_{\mathbf{6}}$ as the 4-dimensional symplectic group over $G F(2)$. Then we may identify $j_{l}=\alpha(l \quad l+1)$ with $(l \quad l+1)\left(\begin{array}{l}7 \\ 8\end{array}\right)$ because there is just one embedding $\mathbf{S}_{\mathbf{6}} \hookrightarrow \mathbf{A}_{\mathbf{8}}$ and $\alpha$ is faithful. Thus (11) may be read in $\mathbf{A}_{\mathbf{8}}$ as $(l-1 l)\left(\begin{array}{ll}7 & 8\end{array}\right)=f_{1}^{-1}(l l+1)\left(\begin{array}{ll}7 & 8\end{array}\right) f_{1}$ and we see that the order of $f_{1}$ should be 6 , a contradiction to (10). Summing up, we have 
3.3.1 Lemma. Let $W$ be a $\mathbf{S}_{m+1}$-invariant $m$-transversal subspace of the vector space associated with $G F(2)^{n} \mathrm{wr}_{\alpha} \mathbf{S}_{m+1}$, where either $m=4$ and $\alpha\left(\mathbf{S}_{\mathbf{4}}\right)=$ $\mathbf{G L}(2,2)$, or $m=5$ and $\alpha\left(\mathbf{S}_{\mathbf{5}}\right)=\mathbf{P} \boldsymbol{\Gamma} \mathbf{L}(2,4)$, or $m=6$ and $\alpha\left(\mathbf{S}_{\mathbf{6}}\right)=\mathbf{S p}(4,2)$. Then $m=5$ and $W$ is the subspace $\sum_{i=1}^{6} f^{i}\left(x_{i}, y_{i}\right)=0$ with $f$ given by $(12)$.

3.4 Consider one of the groups $\mathrm{H}_{t}, t=6,7,8$ or 9 , introduced in 2.4 and 2.5. We ask an $\mathbf{A}_{t}$-invariant $(t-2)$-transversal subspace $W_{t}$ of the vector space $V_{t}$ associated with $\mathrm{H}_{t}$. If we represent each $\mathrm{H}_{t}$ within $\mathrm{H}_{9}$, then $W_{t}$ has the shape

$$
\left\{\left(\sum_{i=1}^{t-2} f_{i}\left(z_{i}\right), \sum_{i=1}^{t-2} g_{i}\left(z_{i}\right), z_{1}, \ldots, z_{t-2}, 0, \ldots, 0\right) \in\left(G F(2)^{4}\right)^{9}: z_{i} \in G F(2)^{4}\right\},
$$

with $f_{i}, g_{i} \in \mathbf{G L}(4,2)$. Such a subspace $W_{t}$ exists and can be described by the following quadratic functions (we regard $V_{9}$ as a vector space over $G F(4)$ ):

$$
\begin{aligned}
& f_{1}(x, y)= \begin{cases}\left(\mathbf{i}^{2} x+\mathbf{i} x^{2}+\mathbf{i}^{2} y+y^{2}, \mathbf{i} x+\mathbf{i}^{2} x^{2}+\mathbf{i}^{2} y+\mathbf{i}^{2} y^{2}\right), & \text { if } t>6, \\
\left(\mathbf{i} x+\mathbf{i}^{2} y, x+y\right), & \text { if } t=6,\end{cases} \\
& g_{1}(x, y)= \begin{cases}\left(\mathbf{i}^{2} x+\mathbf{i} x^{2}+\mathbf{i} y, \mathbf{i}^{2} x+\mathbf{i}^{2} x^{2}+\mathbf{i}^{2} y+\mathbf{i} y^{2}\right), & \text { if } t>6, \\
\left(\mathbf{i} x^{2}+\mathbf{i} y^{2}, \mathbf{i}^{2} y^{2}\right), & \text { if } t=6,\end{cases} \\
& f_{2}=q_{3} f_{1} q^{\prime}, \quad g_{2}=q_{2} g_{1} q^{\prime} \\
& f_{j}=q_{j} f_{j-1} q^{\prime \prime}, \quad g_{j}=q_{j-1} g_{j-1} q^{\prime \prime} \quad(3 \leq j \leq t-2),
\end{aligned}
$$

where $q_{2}, \ldots, q_{t-2}, q^{\prime}, q^{\prime \prime}$ are the functions (7) or (9) according as whether $t=6$ or $t>6$. In fact, one can check that the transformations $Q_{l}$, given by (6), generating a complement of the translation group of $\mathrm{H}_{t}$, leave that subspace invariant.

Actually there are no further possibilities for an $\mathbf{A}_{t}$-invariant $(t-2)$-transversal subspace of $V_{t}$. In order to verify this claim, ask the subspace (13), written over $G F(4)$, to be stable under each transformation (6) for $l=2, \ldots, t-1$, or ask

$$
Q_{l}\left(f_{i}\left(z_{i}\right), g_{i}\left(z_{i}\right), 0, \ldots, 0, z_{i}, 0, \ldots, 0\right) \in W_{t}
$$

for all $z_{i}=\left(x_{i}, y_{i}\right) \in G F(4)^{2}$. In particular we have
a) $f_{1} q^{\prime \prime-1} g_{1}=q^{\prime} q^{\prime \prime}$
$\begin{array}{ll} & (i=1, l=2) \\ g_{1} q^{\prime-1} g_{1}=g_{2} q^{\prime \prime-1} \quad(i=1, l=3)\end{array}$
b) $f_{1} q^{\prime-1} g_{1}+f_{2} q^{\prime \prime-1}=q_{2} f_{1}$,
c) $f_{2}=q_{3} f_{1} q^{\prime}$,
$g_{2}=q_{2} g_{1} q^{\prime} \quad(i=1, l=4)$,
$q_{l-2} g_{1}=g_{1} q_{l-3}$
$(i=1, l=5, \ldots, t-1)$,
d) $q_{l-1} f_{1}=f_{1} q_{l-3}$,
e) $f_{1} q^{\prime-1} g_{2}=q_{2} f_{2}$

with $q_{s}$ from (7) if $t=6$ and from (9) if $t>6$. If $t=6$, then (16d) give

$$
\begin{aligned}
& f_{1}(x, y)=\left(a x+b x^{2}+a^{\prime} y+b^{\prime} y^{2}, \mathbf{i}^{2} a x+\mathbf{i} b x^{2}+\mathbf{i} a^{\prime} y+\mathbf{i}^{2} b^{\prime} y^{2}\right), \\
& g_{1}(x, y)=\left(c x+d x^{2}+c^{\prime} y+d^{\prime} y^{2}, \mathbf{i} c x+\mathbf{i} d^{\prime} y^{2}\right) .
\end{aligned}
$$


From (16b) we infer just two possibilities for $g_{1}$ : either $c=c^{\prime} \neq 0=d$ and $d^{\prime}=\mathbf{i}$, or $c=c^{\prime}=0$ and $d=d^{\prime}=\mathbf{i}$. Assume the first one holds. Then (16a) gives $a=\mathbf{i}, b=c^{2}, a^{\prime}=0, b^{\prime}=\mathbf{i}^{2} c^{2}$, but such a solution does not satisfy (16b). Thus $c=c^{\prime}=0, d=d^{\prime}=\mathbf{i}$ and (16a) says that the functions (14) are the unique possible instances giving an invariant subspace such as (13).

Let $t>6$. Then Equations $(16 d)$ force $f_{1}$ and $g_{1}$ to take the shape

$$
\begin{aligned}
f_{1}(x, y)= & \left(a x+b x^{2}+(\mathbf{i} a+b) y+\mathbf{i}^{2} b y^{2}, \mathbf{i}^{2} b^{2} x+\mathbf{i} a^{2} x^{2}+b^{2} y+\left(a^{2}+\mathbf{i} b^{2}\right) y^{2}\right), \\
g_{1}(x, y)= & \left(c x+d x^{2}+\left(\mathbf{i} c+\mathbf{i}^{2} c^{2}+d\right) y+\left(c+\mathbf{i}^{2} d+\mathbf{i}^{2} d^{2}\right) y^{2},\right. \\
& \left.\left(\mathbf{i} c+\mathbf{i}^{2} d^{2}\right) x+\left(\mathbf{i}^{2} c^{2}+d\right) x^{2}+\left(\mathbf{i}^{2} c+\mathbf{i} c^{2}+\mathbf{i}^{2} d+d^{2}\right) y+\left(\mathbf{i} c^{2}+\mathbf{i}^{2} d\right) y^{2}\right) .
\end{aligned}
$$

By (16a) we have $c=\mathbf{i}\left(a+b+b^{2}\right)$ and $d=\mathbf{i} a^{2}+\mathbf{i} b+\mathbf{i}^{2} b^{2}$, whereas (16c) and (16e) give $f_{1} q^{\prime-1} q_{2} g_{1}=q_{2} q_{3} f_{1}$. This means either $b=1+\mathbf{i} a+\mathbf{i} a^{2}$, or $a=\mathbf{i}^{2}$ and $b=\mathbf{i}$. But using (16b) we see that just the latter can occur. Hence we have

3.4.1 Lemma. The subspace $W_{t}$, defined through (13) and the functions (14), is the unique $\mathbf{A}_{t}$-invariant $(t-2)$-transversal subspace of the vector space $V_{t}$ associated with $\mathrm{H}_{t}(t=6,7,8$ or 9$)$.

Now, we look for an $\mathbf{A}_{t}$-invariant $(t-3)$-transversal subspace $W$ of $V_{t}, t>6$. Within $V_{9}$ we can represent $W$ as

$$
\left\{\left(\sum_{i=1}^{t-3} f_{i}\left(z_{i}\right), \sum_{i=1}^{t-3} g_{i}\left(z_{i}\right), \sum_{i=1}^{t-3} h_{i}\left(z_{i}\right), z_{1}, \ldots, z_{t-3}, 0, \ldots, 0\right) \in\left(G F(2)^{4}\right)^{9}: z_{i} \in G F(2)^{4}\right\}
$$

with $f_{i}, g_{i}, h_{i} \in \mathbf{G L}(4,2)$. As above, we have to ask the subspace (17), written over $G F(4)$, to be stable under each transformation (6) for $l=2, \ldots, t-1$, i.e.

$$
Q_{l}\left(f_{i}\left(z_{i}\right), g_{i}\left(z_{i}\right), h_{i}\left(z_{i}\right), 0, \ldots, 0, z_{i}, 0, \ldots, 0\right) \in W \quad \forall z_{i}=\left(x_{i}, y_{i}\right) \in G F(4)^{2} .
$$

It turns out $f_{2}=q_{4} f_{1} q^{\prime}$ and, for $j>2, f_{j}=q_{j+1} f_{j-1} q^{\prime \prime}$. Furthermore, using (18) for $i=1$ and $l=6$, we have $q_{5} f_{1}=f_{1} q_{2}$; this equation forces $f_{1}$ to take the shape $f_{1}(x, y)=\left(a_{11} x+a_{12} x^{2}+a_{21} y+a_{22} y^{2}\right.$,

$\left.\left(\mathbf{i}^{2} a_{11}^{2}+\mathbf{i} a_{12}^{2}+a_{21}^{2}\right) x+\left(a_{11}^{2}+\mathbf{i}^{2} a_{12}^{2}+\mathbf{i} a_{22}^{2}\right) x^{2}+\left(a_{11}^{2}+\mathbf{i} a_{21}^{2}+\mathbf{i} a_{22}^{2}\right) y+\left(\mathbf{i} a_{12}^{2}+a_{21}^{2}+a_{22}^{2}\right) y^{2}\right)$.

Also, for $i=4$ and $l=5$, we have $q_{4} f_{4}=f_{4} q_{t-2}$, hence $q_{4} f_{4}(1,0)=f_{4} q_{t-2}(1,0)$, giving in turn $a_{21}=\mathbf{i} a_{11}$ and $a_{22}=\mathbf{i}^{2} a_{12}$ for $t=7, a_{12}=a_{11}$ and $a_{22}=\mathbf{i}^{2} a_{11}+\mathbf{i} a_{21}$ for $t=8$. Let $t=7$, then from $q_{4} f_{4}(0,1)=f_{4} q_{5}(0,1)$ we infer $a_{12}=\mathbf{i}^{2} a_{11}$ and $a_{11}=0$. Let $t=8$, then from $q_{4} f_{4}\left(\mathbf{i}^{2}, 0\right)=f_{4} q_{6}\left(\mathbf{i}^{2}, 0\right)$ it follows $a_{21}=\mathbf{i} a_{11}$ and $a_{11}=0$. In both cases we get a contradiction to the fact that $f_{1} \in \mathbf{G L}(4,2)$.

Let now $t=9$. Using (18) for $i=1$ and $l=7$ we obtain $q_{6} f_{1}=f_{1} q_{3}$, which gives $a_{21}=\mathbf{i} a_{11}+a_{11}^{2}+\mathbf{i} a_{12}, \quad a_{22}=\mathbf{i}^{2} a_{11}+\mathbf{i}^{2} a_{12}+\mathbf{i} a_{12}^{2}$, whereas (18) for $i=1$ 
and $l=2$ yields $q^{\prime} q^{\prime \prime} h_{1}=f_{1} q_{7}$ and $q^{\prime-1} f_{1}=g_{1} q_{7}$, hence $q^{\prime} q^{\prime \prime} h_{1}(1,0)=f_{1} q_{7}(1,0)$. As the functions $g_{1}$ and $h_{1}$ fulfill the same conditions (14) as the functions $f_{1}$ and $g_{1}$ defining the subspace $W_{8}$ introduced above, we obtain $a_{11}=a_{12}=1$. But $f_{1}(1,0)=\left(0, \mathbf{i}^{2}\right)$ and $q^{\prime} g_{1} q_{7}(1,0)=(1,1)$, so we get again a contradiction.

Summing up, we have the following

3.4.2 Lemma. For $t=7,8$ or 9 there is no $\mathbf{A}_{t}$-invariant $(t-3)$-transversal subspace in the vector space associated with the group $\mathrm{H}_{t}$ introduced in 2.5.

4.1 Imprimitive groups $m$-transitive on blocks. In the remaining part of this paper, $\mathrm{G}=(G, \Omega)$ will be a permutation group endowed with a finite translation system of imprimitivity $\bar{\Omega}=\left\{\Delta_{1}, \ldots, \Delta_{t}\right\}$ of dimension $m>3$, with inertial group $N$, such that the stabilizer in $G$ of a block is 2-transitive on it and the factor group $G / N$ acts $m$-transitively on $\bar{\Omega}$. This condition confines $\overline{\mathrm{G}}=(G / N, \bar{\Omega})$ to be either the symmetric group on $m$ or $m+1$ elements, or the alternating group on $m+2$ or $m+3$ elements, or a Mathieu group ([9, p. 68] or $[3])$. As the Mathieu groups have no faithful transitive linear representation ([9, p. 68]), by 2.2.1 we have that only the following cases occur:
a) $\overline{\mathrm{G}} \simeq \mathbf{S}_{m}$
b) $\overline{\mathrm{G}} \simeq \mathbf{S}_{m+1}$;
c) $\overline{\mathrm{G}} \simeq \mathbf{A}_{m+2}$
d) $\overline{\mathbf{G}} \simeq \mathbf{A}_{m+3}$.

4.1.1 Remark. A such result can be obtained by assuming that the stabilizer in $G / N$ of $m$ distinct blocks has odd order.

We shall show that not all the above possibilities occur.

4.1.2 Proposition. If $|\Delta| \leq 3$ then either $\overline{\mathrm{G}} \simeq \mathbf{S}_{m}$ or $\overline{\mathrm{G}} \simeq \mathbf{S}_{m+1}$.

Proof. Let $|\Delta|=2$. If $\bar{\Omega}=\left\{\Delta_{1}, \ldots, \Delta_{t}\right\}$, then $\mathrm{G}$ can be embedded into the symmetric group $\mathbf{S}_{2 t}$. Consider distinct non-trivial elements $g_{1}$ and $g_{2}$ in $N$ fixing the points of each of $\Delta_{1}, \ldots, \Delta_{m-2}$. Since $g_{1}$, as well as $g_{2}$, fixes point-wise at most $m-1$ blocks, the fact that each block contains two points implies that the product $g_{1} g_{2}$ leaves at least $t-2$ blocks point-wise fixed. It follows either $t=m$ or $t=m+1$, which means either $\overline{\mathrm{G}} \simeq \mathbf{S}_{m}$ or $\overline{\mathrm{G}} \simeq \mathbf{S}_{m+1}$.

Let $|\Delta|=3$. By theorem 2.2.1, the group $G_{\Delta} / N$ maps homomorphically onto $\mathbb{Z}_{2} \simeq \mathbf{G L}(1,3)$ and this excludes that $\overline{\mathrm{G}}$ is an alternating group.

4.1.3 Theorem. $\bar{G}$ is sharply $m$-transitive.

Proof. We have to rule the possibility $d$ ) out. If $\bar{G} \simeq \mathbf{A}_{m+3}$ then, in view of Theorem 2.2.1, the stabilizer $\mathbf{A}_{m+2}$ has a transitive linear representation on $\Delta$. By [7, Theorem 6.5], if $m+2>8$ then $|\Delta|=m+2$ and $|\mathbf{G L}(\Delta)|<\left|\mathbf{A}_{m+2}\right|$. Hence, since $m>3$, we have $m=4,5$ or 6 and, for each of these values of $m$, just 
one instance occurs $(2.5,[7$, Theorems 5.13, 6.5] or [11]). Hence, according to Theorem 2.2.1, we have that $G$ embeds into one of the groups $\mathbf{H}_{m+3}$ introduced in 2.5 and the vector space $V_{m+3}$ associated with $\mathrm{H}_{m+3}$ has an $\mathbf{A}_{m+3}$-invariant subspace of the shape (17) taking $t=m+3$, but this contradicts Lemma 3.4.2.

4.1.4 Lemma. Let $\overline{\mathrm{G}} \simeq \mathbf{S}_{m}$. Then $\mathrm{G}=G F(p)^{n} \operatorname{wr}_{\alpha} \mathbf{S}_{m}$, where $\alpha$ is one of the following $n$-dimensional transitive linear representations of $\mathbf{S}_{m-1}$ :

1. The trivial representation of $\mathbf{S}_{m-1}$ on a vector line over $G F(2)$;

2. The non-trivial representation of $\mathbf{S}_{m-1}$ on a vector line over $G F(3)$;

3. The faithful representation of $\mathbf{S}_{\mathbf{3}}$ as $\mathbf{G L}(2,2)$;

4. The non-faithful representation of $\mathbf{S}_{\mathbf{4}}$ as $\mathbf{G L}(2,2)$;

5. The representation of $\mathbf{S}_{\mathbf{5}}$ over $G F(2)$ corresponding to $\mathbf{P} \mathbf{\Gamma L}(2,4)$;

6. The representation of $\mathbf{S}_{\mathbf{6}}$ as $\mathbf{S p}(4,2)$.

In any case $G$ splits over the inertial group $N$.

Proof. The first part of the assertion and the fact that $\alpha$ is a transitive linear representation follow from Theorem 2.2.1. If the kernel of the representation $\alpha$ contains $\mathbf{A}_{m-1}$ then $n=1$ and $p=2$ or 3 .

Now we assume that $|\Delta|>3$. Theorem 6.5 in [7] yields $m-1 \leq 6$. Namely, if $m-1>8$ then $|\mathbf{G L}(\Delta)|<\left|\mathbf{S}_{m-1}\right|$, whereas if $m-1 \in\{7,8\}$ we have that $|\Delta|=16$, but $\mathbf{G L}(4,2) \simeq \mathbf{A}_{8}$ and $\mathbf{S}_{7}$ is not contained in $\mathbf{A}_{8}$. The transitive linear representations $\mathbf{3}$ till $\mathbf{6}$ exist (3.3). These are, according to [7, Corollary 5.6 and Theorems 5.12, 5.13] or [11], all transitive linear representations for the groups $\mathbf{S}_{m-1}$, with $4 \leq m \leq 7$.

When $\bar{G}$ is isomorphic to $\mathbf{S}_{m+1}$ or to $\mathbf{A}_{m+2}$ the situation is more complicated. First we shall deal with the case $|\Delta|=2,3$.

4.2 The case $|\Delta|=2$. By Proposition 4.1.2 and Lemma 4.1.4, we may assume that $\bar{G} \simeq \mathbf{S}_{m+1}$. Hence $\mathbf{G}$ can be embedded into the symmetric group $\mathbf{S}_{2(m+1)}$. More precisely, $G$ is a subgroup of order $2^{m}(m+1)$ ! of the subgroup $F$ of order $2^{(m+1)}(m+1)$ ! preserving $\bar{\Omega}$. Then $G$ has index 2 in $F$ and the inertial group $N$ index 2 in the subgroup $M$ of $F$ leaving each block in $\bar{\Omega}$ stable.

From 1.1.4 it follows that the group $G$ contains exactly two involutions leaving the blocks $\Delta_{2}, \ldots, \Delta_{m-1}$ point-wise fixed and switching $\Delta_{m}$ with $\Delta_{m+1}$ and, also, such involutions are conjugate in $G$, hence their product is the non-trivial element of the subgroup $N^{m}$ defined in 1.1.3. By permuting the blocks, we infer that $N$ 
is contained in the subgroup $H$ of $G$ generated by all involutions fixing pointwise $m-2$ blocks and switching other two blocks. Since these involutions induce all transpositions in $G / N \simeq \mathbf{S}_{m+1}$, we obtain $H=G$. As $F$ contains just two distinct classes of conjugate involutions satisfying the above conditions, it follows that there exist at most two distinct permutation groups $(G, \Omega)$. We can actually realize two non-isomorphic permutation groups as follows.

Fix points $X_{1}, \ldots, X_{m+1}$ in $\Omega$, with $X_{i} \in \Delta_{i}$, and identify them with the vectors of a basis $\mathcal{B}$ of a vector space of dimension $m+1$ over $G F(3)$. Also, identify the other point in the block containing $X_{i}$ with $-X_{i}$. This means that we may regard $F$ as the group of permutation matrices in $\mathbf{G L}(m+1,3)$ having the non-zero entries equal to 1 or -1 . Then $N$ is a subgroup of index 2 in the group $M$ of diagonal matrices in $\mathbf{G L}(m+1,3)$. Since there is no element $\neq 1$ in $N$ leaving $m$ blocks point-wise fixed, from 1.1.3 it follows that $N$ consists of all diagonal matrices with determinant 1 .

Let $S$ be the stabilizer in $F$ of the basis $\mathcal{B}$; clearly $S \simeq \mathbf{S}_{m+1}$. Now, look at the following subgroups of index 2 of $F$ :

$$
G_{1}:=N \rtimes S, \quad G_{2}:=\{A \in F: \operatorname{det} A=1\} .
$$

The permutation groups $\left(G_{1}, \Omega\right)$ and $\left(G_{2}, \Omega\right)$ are not isomorphic. In fact, the involution of $G_{1}$ fixing $X_{2}, \ldots, X_{m-1}$ and switching $X_{m}$ with $X_{m+1}$, leaves the block $\Delta_{1}$ pointwise fixed, whereas the involution of $G_{2}$ fulfilling the same conditions acts non-trivially on $\Delta_{1}$. In view of Lemma 4.1.4, the discussion yields

4.2.1 Theorem. Let $\mathcal{B}=\left\{e_{1}, \ldots, e_{m+1}\right\}$ be a basis of a vector space $V$ of dimension $m+1$ over the prime field $G F(3)$ and denote by $N$ the group of linear transformations represented, with respect to $\mathcal{B}$, by diagonal matrices with determinant 1 . If $|\Delta|=2$ then $(G, \Omega)$ is isomorphic to one of the following:

1. $\Omega=\left\{e_{1},-e_{1}, \ldots, e_{m+1},-e_{m+1}\right\}, \quad G=G_{1}=N \rtimes G L(V)_{\mathcal{B}}$;

2. $\Omega=\left\{e_{1},-e_{1}, \ldots, e_{m+1},-e_{m+1}\right\}, \quad G=G_{2}=\left\{g \in G L(V)_{\Omega}: \operatorname{det} g=1\right\}$;

3. $\Omega=\left\{e_{1},-e_{1}, \ldots, e_{m},-e_{m}\right\}, \quad G=G_{3}=G L(V)_{\Omega} \simeq G F(2) \mathrm{wr} \mathbf{S}_{m}$.

Moreover, no two of $G_{1}, G_{2}, G_{3}$ are isomorphic as permutation groups.

Remark. In spite of Theorem 4.2.1, if $m$ is even, the map $g \mapsto(\operatorname{det} g) g$ defines an isomorphism $G_{1} \rightarrow G_{2}$ of abstract groups. But if $m$ is odd, there is no complement of $N$ in $G_{2}$. In order to prove this fact, consider $\varepsilon=\operatorname{diag}(1, \ldots, 1,-1) \in M \backslash N$. The set $\left\{s \varepsilon^{\frac{1}{2}(1-\operatorname{det} s)}: s \in S\right\}$ is a set of representatives of $G_{2} / N$. Furthermore we have

$$
s^{\prime} \varepsilon^{\frac{1}{2}\left(1-\operatorname{det} s^{\prime}\right)} s^{\prime \prime} \varepsilon^{\frac{1}{2}\left(1-\operatorname{det} s^{\prime \prime}\right)}=s^{\prime} s^{\prime \prime} \varepsilon^{\frac{1}{2}\left(2-\operatorname{det} s^{\prime}-\operatorname{det} s^{\prime \prime}\right)} \kappa\left(s^{\prime}, s^{\prime \prime}\right)=s^{\prime} s^{\prime \prime} \varepsilon^{\frac{1}{2}\left(1-\operatorname{det}\left(s^{\prime} s^{\prime \prime}\right)\right)} \kappa\left(s^{\prime}, s^{\prime \prime}\right)
$$

for all $s^{\prime}, s^{\prime \prime} \in S$, where $\kappa: S \times S \rightarrow N$ is the mapping

$$
\left(s^{\prime}, s^{\prime \prime}\right) \longmapsto \begin{cases}s^{\prime \prime-1} \varepsilon s^{\prime \prime} \varepsilon & \text { if } \operatorname{det} s^{\prime}=-1 ; \\ 1_{N} & \text { if } \operatorname{det} s^{\prime}=1 .\end{cases}
$$


This means (see for instance [8, Theorem 17.2]) that $N$ possesses a complement in $G_{2}$ just if there exists a function $\varphi: S \rightarrow N$ such that

$$
\kappa\left(s^{\prime}, s^{\prime \prime}\right)=s^{\prime} \varphi\left(s^{\prime \prime}\right) s^{\prime-1} \varphi\left(s^{\prime}\right) \varphi\left(s^{\prime} s^{\prime \prime}\right)
$$

for all $s^{\prime}, s^{\prime \prime} \in S$. As every element in $N$ has order 2 , the required condition $\kappa\left(1_{S}, 1_{S}\right)=$ $1_{N}$ yields $\varphi\left(1_{S}\right)=1_{N}$. For all $s \in S$, set $\varphi(s)=\operatorname{diag}\left(\varphi_{1}(s), \ldots, \varphi_{m+1}(s)\right)$ and, for $i=1, \ldots, m+1$, put $e_{s(i)}:=s\left(e_{i}\right) \in \mathcal{B}$. Thus we have

$$
\kappa\left(s^{\prime}, s^{\prime \prime}\right)=\operatorname{diag}\left(\ldots \varphi_{s^{\prime-1}(i)}\left(s^{\prime \prime}\right) \varphi_{i}\left(s^{\prime}\right) \varphi_{i}\left(s^{\prime} s^{\prime \prime}\right) \ldots\right) .
$$

Consequently, the product $\varphi_{s^{-1}(i)}\left(s^{\prime \prime}\right) \varphi_{i}\left(s^{\prime}\right) \varphi_{i}\left(s^{\prime} s^{\prime \prime}\right)$ must be 1 if $\operatorname{det} s^{\prime}=1$, or if $s^{\prime \prime}$ leaves the vector $e_{m+1}$ fixed. Otherwise

$$
\varphi_{s^{\prime-1}(i)}\left(s^{\prime \prime}\right) \varphi_{i}\left(s^{\prime}\right) \varphi_{i}\left(s^{\prime} s^{\prime \prime}\right)= \begin{cases}-1 & \text { if } i=m+1, \text { or } s^{\prime \prime}(i)=m+1, \\ +1 & \text { if } i \neq m+1 \neq s^{\prime \prime}(i) .\end{cases}
$$

In particular, if $s$ is an element in $S$ of order 2, we have

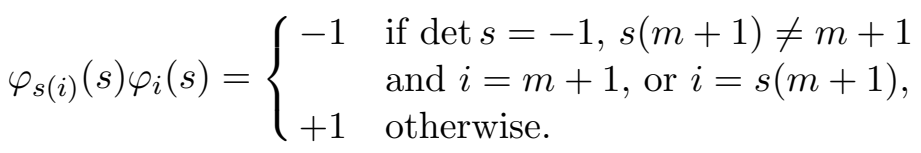

Denote by $\sigma_{i, j}$ the element in $S$ switching the vectors $e_{i}$ and $e_{j}$ and leaving the remaining vectors in $\mathcal{B}$ fixed. Then $(20)$ gives $\varphi_{m}\left(\sigma_{m, m+1}\right) \varphi_{m+1}\left(\sigma_{m, m+1}\right)=-1$. As a consequence, $\varphi\left(\sigma_{m, m+1}\right) \in N$ forces

$$
\varphi_{1}\left(\sigma_{m, m+1}\right) \ldots \varphi_{m-1}\left(\sigma_{m, m+1}\right)=-1 .
$$

Since $m$ is odd, the product $\sigma:=\sigma_{1,2} \ldots \sigma_{m-2, m-1}$ is well-defined. The relations $\sigma\left(e_{m+1}\right)=e_{m+1}$ and $\sigma^{2}=1_{S}$ give

$$
\varphi_{1}(\sigma) \varphi_{2}(\sigma)=\ldots=\varphi_{m-2}(\sigma) \varphi_{m-1}(\sigma)=1 .
$$

If 4 would not divide $m+1$, then $\operatorname{det}\left(\sigma_{m, m+1} \sigma\right)=-1$ which means

$$
\begin{aligned}
1 & =\varphi_{i}\left(\sigma_{m, m+1} \sigma\right) \varphi_{i+1}\left(\sigma_{m, m+1} \sigma\right) \quad(i=1, \ldots, m-2), \\
-1 & =\varphi_{m}\left(\sigma_{m, m+1} \sigma\right) \varphi_{m+1}\left(\sigma_{m, m+1} \sigma\right)
\end{aligned}
$$

i.e. $\varphi\left(\sigma_{m, m+1} \sigma\right) \notin N$. Therefore 4 divides $m+1$ and we have

$$
\varphi_{1}\left(\sigma_{m, m+1} \sigma\right) \varphi_{2}\left(\sigma_{m, m+1} \sigma\right)=\ldots=\varphi_{m}\left(\sigma_{m, m+1} \sigma\right) \varphi_{m+1}\left(\sigma_{m, m+1} \sigma\right)=1 .
$$

Use now Equations (19) for $s^{\prime}=\sigma$ and $s^{\prime \prime}=\sigma_{m, m+1}$. It follows

$$
\varphi_{2}\left(\sigma_{m, m+1}\right) \varphi_{1}(\sigma) \varphi_{1}\left(\sigma_{m, m+1} \sigma\right)=\varphi_{1}\left(\sigma_{m, m+1}\right) \varphi_{2}(\sigma) \varphi_{2}\left(\sigma_{m, m+1} \sigma\right)=1 .
$$

Therefore $\varphi_{1}\left(\sigma_{m, m+1}\right) \varphi_{2}\left(\sigma_{m, m+1}\right)=1$ because we know that $\varphi_{1}(\sigma) \varphi_{2}(\sigma)=1$ and $\varphi_{1}\left(\sigma_{m, m+1} \sigma\right) \varphi_{2}\left(\sigma_{m, m+1} \sigma\right)=1$. Likewise, we get

$$
\varphi_{3}\left(\sigma_{m, m+1}\right) \varphi_{4}\left(\sigma_{m, m+1}\right)=\ldots=\varphi_{m-2}\left(\sigma_{m, m+1}\right) \varphi_{m-1}\left(\sigma_{m, m+1}\right)=1,
$$

a contradiction to $(21)$. 
4.3 The case $|\Delta|=3$. By Proposition 4.1.2 and Lemma 4.1.4 we may assume that $\overline{\mathrm{G}} \simeq \mathbf{S}_{m+1}$. According to Theorem 2.2.1, we may identify $\mathrm{G}$ with a subgroup of the twisted wreath product $\tilde{\mathrm{G}}=G F(3) \mathrm{wr}_{\alpha} \mathbf{S}_{m+1}$, where $\alpha$ is the non-trivial linear representation of $\mathbf{S}_{m}$ on the 1-dimensional vector space over $G F(3)$; then, the inertial group $N$ is defined by a $\mathbf{S}_{m+1}$-invariant $m$-transversal subspace $W$ of the vector space $V$ associated with $\tilde{\mathrm{G}}$, which is a hyperplane (Lemma 3.2.1).

Let $S \simeq \mathbf{S}_{m+1}$ be a translation complement in $\tilde{\mathrm{G}}$ and let $A$ be the subgroup of $S$ isomorphic to $\mathbf{A}_{m+1}$. Consider in the group $\tilde{G} / N \simeq(V / N)>\triangleleft(S N / N)$ the subgroup $(V / N)>\triangleleft(A N / N)$. As $V / N$ is 1-dimensional, the last semi-direct product must be direct. Then we can use the subsequent lemma to conclude that $A N=\varphi^{-1}(A N / N)$, where $\varphi: G \rightarrow G / N$ denotes the canonical homomorphism.

4.3.1 Lemma. Let $G=V>\triangleleft A$ be a semidirect product of an solvable group $V$ by a group $A$ isomorphic to the alternating group $\mathbf{A}_{t}, t>4$. Besides let $N$ be a normal subgroup of $G$ contained in $V$ and let $H$ be a subgroup of $G$ containing $N$ such that $H / N \simeq A$. If $A N / N$ centralizes $V / N$, then $H=N A$.

Proof. We may regard $H / N$ as a subgroup of the direct product $(V / N) \times(A N / N)$, so $H / N$ projects into both $V / N$ and $A N / N$. As $V / N$ is solvable, the first projection is trivial; hence $H / N=A N / N$, i.e. $H=A N$.

Now, since $A N$ has index 2 in $G$, we see that $G$ splits over $N$ by a theorem of Gaschütz ([8, p. 121]). Thus, in view of 2.2.1, 3.2 and 4.1.4, we can state the following

4.3.2 Theorem. Let $|\Delta|=3$; then we have $\overline{\mathrm{G}} \simeq \mathbf{S}_{t}$, where $t=m$ or $t=m+1, G$ splits over the inertial group $N$ and $\mathrm{G}$ can be realized as follows:

Let $V$ be the vector space of dimension $t$ over the prime field $G F(3)$ and let $\Delta_{i}=$ $\left\{x+V_{i}: x \in V\right\}$, where $V_{i}$ denotes the hyperplane of vectors $\left(x_{1}, \ldots, x_{t}\right)$ with $x_{i}=0$. Then, $\Omega=\Delta_{1} \cup \ldots \cup \Delta_{t}$ and $G=\{x \mapsto w+g(x): w \in W, g \in S\}$, where $W=V$ if $t=m, W=\left\{\left(x_{1}, \ldots, x_{t}\right) \in V: \sum_{k=1}^{m+1}(-1)^{m(k+1)} x_{k}=0\right\}$ if $t=m+1$, and $S$ denotes the subgroup of $\mathbf{G L}(V)$ generated by the linear mappings

$$
\begin{aligned}
\left(x_{1}, \ldots, x_{t}\right) & \mapsto\left(x_{t}, x_{1}, \ldots, x_{t-1}\right), \\
\left(x_{1}, \ldots, x_{t}\right) & \mapsto\left((-1)^{t} x_{t},-x_{2}, \ldots,-x_{t-1},(-1)^{t} x_{1}\right),
\end{aligned}
$$

corresponding to the permutations $(1, \ldots, t)$ and $(1 t)$, respectively.

In view of Theorems 4.2.1 and 4.3.2 from now on we may assume $|\Delta|>3$. Before proceeding, we need to state the following general lemma.

4.3.3 Lemma. Let $V$ be a $G F(2)$-vector space, $S=\left\langle h_{i}: i \in I\right\rangle$ be a group of automorphisms of $V$ generated by elements $h_{i}$ of prime order $p_{i}$ leaving a proper subspace $W$ of $V$ invariant and $0_{V} \longrightarrow W \longrightarrow G \stackrel{\lambda}{\longrightarrow} S \longrightarrow 1_{S}$ be an extension of $W$ by $S$. Assume $\operatorname{ker}\left(i d_{W}+h_{i \mid W}\right) \subseteq \operatorname{im}\left(i d_{W}+h_{i \mid W}\right)$ if $p_{i}=2$. Then the following hold:

$$
0_{V} \longrightarrow W \longrightarrow G \longrightarrow \lambda S \longrightarrow 1_{S}
$$


a) For all $k \in I$ there exists $g_{k} \in G$ such that $\lambda\left(g_{k}\right)=h_{k}$ and $g_{k}^{p_{k}}=1_{G}$.

b) Let $h_{i}$ and $h_{j}$ be of order 2 with $\left(h_{i} h_{j}\right)^{3}=1_{S}$. Then, for $k=i, j$, there exists $g_{k} \in G$ such that $\lambda\left(g_{k}\right)=h_{k}$ and $g_{k}^{2}=\left(g_{i} g_{j}\right)^{3}=1_{G}$.

c) Let $h_{i}$ and $h_{j}$ be of order 2 with $\left(h_{i} h_{j}\right)^{2}=1_{S}$. Then, for $k=i, j$, there exists $g_{k} \in G$ such that $\lambda\left(g_{k}\right)=h_{k}, g_{k}^{2}=1_{G}$ and $\left(g_{i} g_{j}\right)^{2} \in \operatorname{Fix}\left(h_{k}\right)$.

d) Let $h_{i}$ and $h_{j}$ be of order 2 and 3 , respectively, with $\left(h_{i} h_{j}\right)^{2}=1_{S}$. Then, for $k=i, j$, there exists $g_{k} \in G$ such that $\lambda\left(g_{k}\right)=h_{k}, g_{i}^{2}=g_{j}^{3}=1_{G}$ and $\left(g_{i} g_{j}\right)^{2} \in$ $\operatorname{Fix}\left(h_{i} h_{j}\right) \cap \operatorname{Fix}\left(h_{j}+h_{j}^{2}\right)$.

Proof. a) Let $g_{k} \in \lambda^{-1}\left(h_{k}\right)$, then $g_{k}$ centralizes $g_{k}^{p_{k}}=w_{k} \in W$. If $p_{k}$ is odd, $\left(w_{k} g_{k}\right)^{p_{k}}=\left(w_{k}\right)^{p_{k}}\left(g_{k}\right)^{p_{k}}=w_{k}+w_{k}=0_{W}$. If $p_{k}=2$ then $h_{k}\left(w_{k}\right)=g_{k} w_{k} g_{k}^{-1}=w_{k}$, hence $w_{k}=h_{k}\left(u_{k}\right)+u_{k}$ for some $u_{k} \in W$ and we have $\left(u_{k} g_{k}\right)^{2}=u_{k} g_{k} u_{k} g_{k}^{-1} g_{k}^{2}=$ $u_{k}+h_{k}\left(u_{k}\right)+w_{k}=0_{W}$.

b) Take $g_{k}$ as in $\left.a\right)$, thus $g_{k}(x)+h_{k}(x)$ is a well-defined vector $u_{k}$ for all $x \in V$. Put $w_{i j}=\left(g_{i} g_{j}\right)^{3}(\in W)$, then $g_{k}^{2}=1_{G}$ gives $h_{k}\left(u_{k}\right)=u_{k}$, whereas $\left(h_{i} h_{j}\right)^{3}=1_{S}$ yields

$$
w_{i j}=h_{i} h_{j}\left(u_{i}\right)+h_{j} h_{i}\left(u_{j}\right)+h_{i}\left(u_{j}\right)+h_{j}\left(u_{i}\right)+u_{i}+u_{j} .
$$

Thus $h_{k}\left(w_{i j}\right)=w_{i j}$ and we see that $\left(w_{i j} g_{i} g_{j}\right)^{3}=\left(w_{i j} g_{i}\right)^{2}=1_{G}$.

$c)$ Take $g_{k}$ as in $\left.a\right)$ and put $v_{i j}=\left(g_{i} g_{j}\right)^{2}(\in W)$. Then $g_{i} g_{j} g_{i} g_{j}=g_{j} g_{i} g_{j} g_{i}$ and we see that $h_{k}$ leaves $v_{i j}$ fixed.

d) Taking $g_{k}$ as in $a$ ), $u_{k}$ as in the proof of $b$ ) and $v_{i j}$ as in the proof of $c$ ), we have $h_{i} h_{j}\left(v_{i j}\right)=v_{i j}$. Furthermore $g_{i}^{2}=g_{j}^{3}=1_{G}$ give $h_{i}\left(u_{i}\right)=u_{i}$ and $\left(h_{j}+h_{j}^{2}\right)\left(u_{j}\right)=u_{j}$, whereas $\left(g_{i} g_{j}\right)^{2}=1_{G}$ implies $v_{i j}=h_{j}^{2}\left(u_{i}+u_{j}\right)+h_{i}\left(u_{j}\right)+u_{i}$. So $\left(h_{j}+h_{j}^{2}\right)\left(v_{i j}\right)=v_{i j}$.

4.4 The case $\bar{G} \simeq \mathbf{S}_{m+1}$. As $|\Delta|>3$, the permutation group $\mathrm{G}$ embeds into $\tilde{\mathrm{G}}=G F(p)^{n} \operatorname{wr}_{\alpha} \mathbf{S}_{m+1}$ with $\alpha$ one of the representations $\mathbf{4}, \mathbf{5}$ or $\mathbf{6}$ given in Lemma 4.1.4. Also, thanks to Lemma 3.3.1, we have $m=5$ and the translations in the inertial group $N$ are defined through vectors in the subspace

$$
W=\left\{\left(z_{1}, \ldots, z_{6}\right) \in V=\left(G F(4)^{2}\right)^{6}: \sum_{i=1}^{6} f^{i}\left(z_{i}\right)=0\right\},
$$

where $f: G F(4)^{2} \longrightarrow G F(4)^{2}$ is the bijective mapping defined by

$$
(x, y) \longmapsto\left(\mathbf{i} x+\mathbf{i} y+\mathbf{i} y^{2}, \mathbf{i}^{2} x+\mathbf{i}^{2} y^{2}\right) .
$$

So $G$ embeds into the group $G_{6}$ introduced in 2.3 and we have to determine the nonequivalent extensions within $\mathrm{G}_{6}$ of $N$ by the group $S$ generated by the matrices (3), with $k=1, \ldots, 5$ and entries (4) and (5). It turns out that $\operatorname{ker}\left(J_{k \mid W}+i d_{W}\right)=$ $\operatorname{im}\left(J_{k \mid W}+i d_{W}\right)$ so Lemma 4.3.3, $a$ applies and we find in $G$ elements

$$
g_{k}: v \mapsto J_{k}(v)+u_{k} \quad(k=1, \ldots, 5)
$$

of order 2. Then $u_{k} \in \operatorname{Fix}\left(J_{k}\right)$ and we have 


$$
\begin{aligned}
& u_{1}=\left(\mathbf{i} x_{1}+\mathbf{i} y_{1}, \mathbf{i}^{2} x_{1}, x_{1}, y_{1}, z_{1}, z_{1}^{2}, t_{1}, t_{12}, \mathbf{i} r_{11}, \mathbf{i}^{2} r_{12}, s_{11}, s_{12}\right), \\
& u_{2}=\left(s_{21}, s_{22}, \mathbf{i} x_{2}+\mathbf{i} y_{2}, \mathbf{i}^{2} x_{2}, x_{2}, y_{2}, z_{2}, z_{2}^{2}, t_{2}, t_{22}, \mathbf{i} r_{21}, \mathbf{i}^{2} r_{22}\right), \\
& u_{3}=\left(\mathbf{i} r_{31}, \mathbf{i}^{2} r_{32}, s_{31}, s_{32}, \mathbf{i} x_{3}+\mathbf{i} y_{3}, \mathbf{i}^{2} x_{3}, x_{3}, y_{3}, z_{3}, z_{3}^{2}, t_{3}, t_{32}\right), \\
& u_{4}=\left(t_{4}, t_{42}, \mathbf{i} r_{41}, \mathbf{i}^{2} r_{42}, s_{41}, s_{42}, \mathbf{i} x_{4}+\mathbf{i} y_{4}, \mathbf{i}^{2} x_{4}, x_{4}, y_{4}, z_{4}, z_{4}^{2}\right), \\
& u_{5}=\left(z_{5}, z_{5}^{2}, t_{5}, t_{52}, \mathbf{i} r_{51}, \mathbf{i}^{2} r_{52}, s_{51}, s_{52}, \mathbf{i} x_{5}+\mathbf{i} y_{5}, \mathbf{i}^{2} x_{5}, x_{5}, y_{5}\right),
\end{aligned}
$$

where the entries $r_{k h}, s_{k h}, t_{k h}, x_{k h}, y_{k h}, z_{k h}$ take the value 1 or 0 and $t_{k}=t_{k 1}+\mathbf{i} t_{k 2}, x_{k}=$ $x_{k 1}+\mathbf{i} x_{k 2}, y_{k}=y_{k 1}+\mathbf{i} y_{k 2}, z_{k}=z_{k 1}+\mathbf{i} z_{k 2}$. Notice that $u_{k} \in W$ just if

$$
\begin{aligned}
& \text { a) } y_{k 1}+z_{k 1}+z_{k 2}+t_{k 1}+r_{k 1}+s_{k 1}=0 \\
& \text { b) } y_{k 2}+z_{k 1}+t_{k 2}+r_{k 1}+r_{k 2}+s_{k 2}=0
\end{aligned}
$$

As $\left(J_{l} J_{l-1}\right)^{3}=1_{S}$, we may assume $\left(g_{l} g_{l-1}\right)^{3}=1_{G}$ (Lemma $\left.4.3 .3, b\right)$ which means

$$
J_{l}\left(u_{l-1}\right)+u_{l} \in \operatorname{Fix}\left(Q_{l}^{2}+Q_{l}\right) \quad(l=2, \ldots, 5),
$$

where $Q_{l}=J_{l-1} J_{l}$ are the matrices (3) and (6). The last conditions give

$$
\begin{aligned}
& \text { a) } x_{l-11}+x_{l-12}+y_{l-11}+y_{l-12}+z_{l-11}+z_{l-12}=x_{l 1}+x_{l 2}+y_{l 1}+s_{l 1} \text {, } \\
& \text { b) } x_{l-11}+z_{l-12}=x_{l 2}+y_{l 2}+s_{l 2} .
\end{aligned}
$$

For all $i, j=1, \ldots, 5$ with $|i-j|>1$ we have $J_{i} J_{j}=J_{j} J_{i}$, hence

$$
v_{i j}:=\left(g_{i} g_{j}\right)^{2}(v)+v \in \operatorname{Fix}\left(J_{i}\right) \bigcap \operatorname{Fix}\left(J_{j}\right)
$$

by Lemma 4.3.3,c. Thus the coordinates of $v_{i j}$ have the following shape:

$$
\begin{aligned}
& v_{13}=v_{31}=\left(\mathbf{i} a_{13}+\mathbf{i} b_{13}, \mathbf{i}^{2} a_{13}, a_{13}, b_{13}, c_{13}+\mathbf{i} d_{13}, c_{13}+\mathbf{i}^{2} d_{13},\right. \\
& \left.d_{13}+\mathbf{i} c_{13}, c_{13}, \mathbf{i} e_{13}, \mathbf{i}^{2} e_{13}, b_{13}+c_{13}+e_{13}, 0\right), \\
& v_{15}=v_{51}=\left(c_{15}+\mathbf{i} d_{15}, c_{15}+\mathbf{i}^{2} d_{15}, d_{15}+\mathbf{i} c_{15}, c_{15}, \mathbf{i} e_{15}, \mathbf{i}^{2} e_{15},\right. \\
& \left.b_{15}+c_{15}+e_{15}, 0, \mathbf{i} a_{15}+\mathbf{i} b_{15}, \mathbf{i}^{2} a_{15}, a_{15}, b_{15}\right), \\
& v_{35}=v_{53}=\left(\mathbf{i} e_{35}, \mathbf{i}^{2} e_{35}, b_{35}+c_{35}+e_{35}, 0, \mathbf{i} a_{35}+\mathbf{i} b_{35}, \mathbf{i}^{2} a_{35},\right. \\
& \left.a_{35}, b_{35}, c_{35}+\mathbf{i} d_{35}, c_{35}+\mathbf{i}^{2} d_{35}, d_{35}+\mathbf{i} c_{35}, c_{35}\right), \\
& v_{24}=v_{42}=\left(b_{24}+c_{24}+e_{24}, 0, \mathbf{i} a_{24}+\mathbf{i} b_{24}, \mathbf{i}^{2} a_{24}, a_{24}, b_{24},\right. \\
& \left.c_{24}+\mathbf{i} d_{24}, c_{24}+\mathbf{i}^{2} d_{24}, d_{24}+\mathbf{i} c_{24}, c_{24}, \mathbf{i} e_{24}, \mathbf{i}^{2} e_{24}\right), \\
& v_{14}=v_{41}=\left(\mathbf{i}^{2} a_{14}+b_{14}, a_{14}, \mathbf{i} a_{14}, \mathbf{i}^{2} b_{14}, c_{14}, c_{14}\right. \text {, } \\
& \left.\mathbf{i}^{2} e_{14}+b_{14}+c_{14}+d_{14}, e_{14}, \mathbf{i} e_{14}, \mathbf{i}^{2} b_{14}+\mathbf{i}^{2} c_{14}+\mathbf{i}^{2} d_{14}, d_{14}, d_{14}\right), \\
& v_{25}=v_{52}=\left(c_{25}, c_{25}, \mathbf{i}^{2} e_{25}+b_{25}+c_{25}+d_{25}, e_{25}, \mathbf{i} e_{25}, \mathbf{i}^{2} b_{25}+\mathbf{i}^{2} c_{25}+\mathbf{i}^{2} d_{25}\right. \text {, } \\
& \left.d_{25}, d_{25}, \mathbf{i}^{2} a_{25}+b_{25}, a_{25}, \mathbf{i} a_{25}, \mathbf{i}^{2} b_{25}\right),
\end{aligned}
$$

where the entries $a_{i j}, b_{i j}, c_{i j}, d_{i j}, e_{i j}$ take the value 0 or 1 . Notice that $v_{i j}=0$ precisely if the coordinates of $u_{i}$ and $u_{j}$ satisfy the conditions 

a) $x_{i 1}=t_{j 2}+r_{j 1}, \quad$ b) $x_{i 1}+y_{i 1}+y_{i 2}=t_{j 1}+r_{j 1}+r_{j 2}$,
c) $z_{i 2}=s_{j 1}+s_{j 2}, \quad$ d) $t_{i 1}+t_{i 2}+r_{i 2}=y_{j 1}+y_{j 2}$,
if $j=i+3$,
e) $t_{i 2}+r_{i 1}=x_{j 1}$,
f) $s_{i 1}+s_{i 2}=z_{j 2}$,
g) $x_{i 2}=r_{j 2}+s_{j 1}$
h) $y_{i 2}=r_{j 1}+r_{j 2}+s_{j 2}$,
i) $z_{i 1}+t_{i 2}=y_{j 2}, \quad$ j) $z_{i 2}+t_{i 1}=x_{j 2}+y_{j 1}+y_{j 2}$,
k) $r_{i 1}+r_{i 2}=z_{j 1}, \quad$ l) $s_{i 2}=t_{j 2}$,

In order that the extension exists, the vectors $v_{i j}$ are subject to conditions determined by N. Blackburn in [2, pp. 205-206]. These conditions, which are also sufficient for the existence of the extension, can be written as follows:

$$
\begin{aligned}
& \text { a) }\left(J_{1}+i d_{V}\right)\left(v_{35}\right)+\left(J_{3}+i d_{V}\right)\left(v_{15}\right)+\left(J_{5}+i d_{V}\right)\left(v_{13}\right)=0_{V} \\
& \text { b) }\left(Q_{l}^{2}+Q_{l}+i d_{V}\right)\left(v_{l-1 j}+v_{l j}\right)=0_{V} \\
& \text { c) }\left(Q_{l}^{2}+Q_{l}+i d_{V}\right)\left(Q_{l+1}^{2}+i d_{V}\right)\left(v_{l-1 l+1}\right)=0_{V}
\end{aligned}
$$

where $l$ takes the values $2,3,4,5$ in $b)(j \neq l-2, l-1, l, l+1)$ and $2,3,4$ in $c)$.

Now the aim is to show that we may take zero all the vectors $v_{i j} \neq v_{15}$. We can do this by adding to $u_{k}$ a vector $w_{k} \in W \cap \operatorname{Fix}\left(J_{k}\right)$ with $J_{l}\left(w_{l-1}\right)+w_{l} \in \operatorname{Fix}\left(Q_{l}^{2}+Q_{l}\right)$ $(l=2, \ldots, 5)$. Then we may assume that $v_{i j}=0_{V}$ if $w_{i}$ and $w_{j}$ satisfy the condition $\left(J_{i}+i d_{V}\right)\left(w_{j}\right)=\left(J_{j}+i d_{V}\right)\left(w_{i}\right)$. We shall proceed by annihilating the vectors $v_{i j}$ successively and, then, considering new vectors $w_{k}$ which retain the achieved conditions $v_{i j}=0_{V}$. As a matter of fact, adding to $u_{1}$ the vector

$$
\begin{aligned}
& \left(a_{13}+b_{13}+\mathbf{i} c_{13}+\mathbf{i} d_{13}, a_{13}+\mathbf{i}^{2} d_{13}, d_{13}+\mathbf{i} a_{13}, a_{13}+c_{13}+\mathbf{i}^{2} b_{13}, c_{13}+\mathbf{i} d_{13},\right. \\
& \left.c_{13}+\mathbf{i}^{2} d_{13}, 0,0, \mathbf{i} a_{13}+\mathbf{i} b_{13}+\mathbf{i} d_{13}, \mathbf{i}^{2} a_{13}+\mathbf{i}^{2} b_{13}+\mathbf{i}^{2} d_{13}+\mathbf{i}^{2} e_{13}, 0, b_{13}+c_{13}+e_{13}\right)
\end{aligned}
$$

we make $v_{13}=0$, whereas adding to $u_{2}$ the vector

$$
\begin{gathered}
\left(a_{24}+b_{24}, a_{24}+\mathbf{i}^{2} b_{24}, \mathbf{i} a_{24}+\mathbf{i}^{2}\left(b_{24}+e_{24}\right), b_{24}+e_{24}+\mathbf{i}^{2} a_{24}, e_{24}+\mathbf{i}^{2} b_{24},\right. \\
\mathbf{i} b_{24}+\mathbf{i}^{2}\left(a_{24}+e_{24}\right), \mathbf{i}\left(a_{24}+e_{24}\right)+\mathbf{i}^{2} b_{24}, a_{24}+b_{24}+d_{24}+e_{24}, 0, \\
\left.\mathbf{i}\left(a_{24}+b_{24}+d_{24}\right), \mathbf{i}^{2}\left(a_{24}+b_{24}+d_{24}+e_{24}\right)\right)
\end{gathered}
$$

and to $u_{4}$ the vector

$$
\begin{gathered}
\left(\mathbf{i}^{2}\left(a_{24}+c_{24}+e_{24}\right), a_{24}+c_{24}+e_{24}, 0,0,0,0, \mathbf{i}\left(a_{24}+c_{24}+e_{24}\right), \mathbf{i}\left(a_{24}+c_{24}+e_{24}\right),\right. \\
\left.\mathbf{i}^{2}\left(a_{24}+c_{24}+e_{24}\right), \mathbf{i}\left(a_{24}+c_{24}+e_{24}\right), \mathbf{i}\left(a_{24}+c_{24}+e_{24}\right), \mathbf{i}^{2}\left(a_{24}+c_{24}+e_{24}\right)\right),
\end{gathered}
$$

we make $v_{24}=0$. Also the vector $w_{3}=\mathbf{i}\left(a_{35}, 0,0, a_{35}, a_{35}, a_{35}, \mathbf{i} a_{35}, a_{35}, 0,0,0,0\right)$ is in $\operatorname{Fix}\left(J_{1}\right)$, so replacing $u_{3}$ by $u_{3}+w_{3}$ and $u_{5}$ by $u_{5}+w_{5}$, where $w_{5}$ is the vector

$$
\begin{gathered}
\left(\mathbf{i}\left(b_{35}+d_{35}\right)+\mathbf{i}^{2}\left(a_{35}+e_{35}\right), \mathbf{i}\left(a_{35}+e_{35}\right)+\mathbf{i}^{2}\left(b_{35}+d_{35}\right), \mathbf{i}\left(a_{35}+b_{35}+c_{35}+e_{35}\right),\right. \\
\left.a_{35}+b_{35}+c_{35}+e_{35}, \mathbf{i} b_{35}, 0,0,0, \mathbf{i} c_{35}, \mathbf{i} c_{35}+\mathbf{i}^{2} d_{35}, d_{35}+\mathbf{i}^{2} c_{35}, d_{35}+\mathbf{i} c_{35}\right),
\end{gathered}
$$

we make $v_{35}=0$ without destroying $v_{13}=0$. Notice that $v_{13}=0, v_{24}=0$ and $(26 b)$ 
imply that $v_{14} \in \operatorname{Fix}\left(Q_{2}^{2}+Q_{2}\right) \cap \operatorname{Fix}\left(Q_{4}^{2}+Q_{4}\right)$, which means $c_{14}=a_{14}$ and $e_{14}=b_{14}+d_{14}$. Also, $v_{24}=0_{V}, v_{35}=0_{V}$ and $(26 b)$ give $v_{25} \in \operatorname{Fix}\left(Q_{3}^{2}+Q_{3}\right) \cap \operatorname{Fix}\left(Q_{5}^{2}+Q_{5}\right)$, or $e_{25}=d_{25}=a_{25}+b_{25}$. Thus we can annihilate $v_{14}$ and $v_{25}$ by adding

$$
\begin{aligned}
& w_{1}=\left(\mathbf{i} b_{14}, 0,0, b_{14}, b_{14}, b_{14}, \mathbf{i} b_{14}, b_{14}, 0,0,0,0\right), \\
& w_{4}=\left(d_{14}, 0, \mathbf{i} a_{14}, \mathbf{i}^{2} d_{14}, d_{14}, a_{14}+d_{14}, a_{14}+\mathbf{i}^{2} d_{14}, a_{14}+\mathbf{i} d_{14}, \mathbf{i} a_{14}+\mathbf{i}^{2} d_{14}, a_{14}+d_{14}, \mathbf{i} d_{14}, \mathbf{i}^{2} d_{14}\right), \\
& \left.w_{5}=\mathbf{i} c_{25}, \mathbf{i}^{2} c_{25}, c_{25}, 0, \mathbf{i}\left(a_{25}+b_{25}\right), \mathbf{i}^{2} a_{25}, a_{25}, b_{25}, b_{25}+\mathbf{i} a_{25}, b_{25}+\mathbf{i}^{2} a_{25}, a_{25}+\mathbf{i} b_{25}, b_{25}\right)
\end{aligned}
$$

to $u_{1}, u_{4}$ and $u_{5}$, respectively (notice that $w_{1} \in \operatorname{Fix}\left(J_{3}\right), w_{4} \in \operatorname{Fix}\left(J_{2}\right), w_{5} \in \operatorname{Fix}\left(J_{3}\right)$ ).

Now the question is whether we can annihilate $v_{15}$ or not. Since we are assuming $v_{14}=v_{25}=v_{13}=v_{35}=0_{V}$, from $(26 a)$ and $(26 b)$ it follows that $v_{15}$ is contained in the intersection $\operatorname{Fix}\left(J_{3}\right) \cap \operatorname{Fix}\left(Q_{2}^{2}+Q_{2}\right) \cap \operatorname{Fix}\left(Q_{5}^{2}+Q_{5}\right)$. Therefore,

$$
v_{15}=\left(\mathbf{i} a, \mathbf{i}^{2} a, a, 0, \mathbf{i} a, \mathbf{i}^{2} a, a, 0, \mathbf{i} a, \mathbf{i}^{2} a, a, 0\right)
$$

with $a=0$, or $a=1$. We claim that if $a=1$ no further replacement of the vectors $u_{k}$ by vectors $u_{k}+w_{k}$ gives $v_{15}=0$ (without destroying the achieved conditions). In fact, if such vectors $w_{k}$ exist, they should have coordinates $x_{k h}, y_{k h}, z_{k h}, t_{k h}, r_{k h}, s_{k h}$, written as in (22) for $u_{k}$ and satisfying (23), (24) and (25) (the latter for $(i, j) \neq(1,5)$ ). Also they should fulfill the condition $\left(J_{1}+i d_{V}\right)\left(w_{5}\right)+\left(J_{5}+i d_{V}\right)\left(w_{1}\right)=v_{15}$, i.e., by comparing the last coordinate but one,

$$
r_{12}+s_{11}+x_{52}=1
$$

But (28) is incompatible with the following system of equations

$$
\begin{array}{ll}
r_{11}+y_{11}+z_{11}+z_{12}+t_{11}+s_{11}=0, & (23 a), k=1, \\
r_{21}+y_{21}+z_{21}+z_{22}+t_{21}+s_{21}=0, & (23 a), k=2, \\
r_{31}+y_{31}+z_{31}+z_{32}+t_{31}+s_{31}=0, & (23 a), k=3, \\
r_{22}+y_{22}+z_{21}+t_{22}+r_{21}+s_{22}=0, & (23 b), k=2, \\
x_{11}+x_{12}+y_{11}+y_{12}+z_{11}+z_{12}+x_{21}+x_{22}+y_{21}+s_{21}=0, & (24 a), l=2, \\
x_{11}+z_{12}+x_{22}+y_{22}+s_{22}=0, & (24 b), l=2, \\
x_{21}+z_{22}+x_{32}+y_{32}+s_{32}=0, & (24 b), l=3, \\
t_{21}+t_{22}+r_{22}+y_{51}+y_{52}=0, & (25 d), i=2, j=5, \\
x_{12}+r_{32}+s_{31}=0, & (25 g), i=1, j=3, \\
y_{12}+r_{31}+r_{32}+s_{32}=0, & (25 h), i=1, j=3, \\
z_{12}+t_{11}+x_{32}+y_{31}+y_{32}=0, & (25 j), i=1, j=3, \\
z_{32}+t_{31}+x_{52}+y_{51}+y_{52}=0, & (25 j), i=3, j=5, \\
r_{11}+r_{12}+z_{31}=0, & (25 k), i=1, j=3,
\end{array}
$$

since the left side of (28) is the sum of all the left sides of the above system. This shows that there is just one non-splitting extension: it is achieved by the vectors

$$
\begin{array}{ll}
u_{1}=(0,0,0,0,0,0,0,0,0,0,1,0), & u_{2}=u_{3}=(0,0,0,0,0,0,0,0,0,0,0,0), \\
u_{4}=\left(0,0,0,0,0,0,0,0,0,0, \mathbf{i}, \mathbf{i}^{2}\right), & u_{5}=\left(0,0,1,0,0, \mathbf{i}^{2}, 1,1,0,0,0,0\right) .
\end{array}
$$

In fact, such vectors solve $(22),(24)$ and $(25)$ for $(i, j) \neq(1,5)$; furthermore, the vector 
$\left(J_{1}+i d_{V}\right)\left(u_{5}\right)+\left(J_{5}+i d_{V}\right)\left(u_{1}\right)$ is precisely (27). Summing up we have

4.4.1 Theorem. Let $\overline{\mathrm{G}} \simeq \mathbf{S}_{m+1}$ and assume that the blocks contain more than 3 points. Then $m=5$, the blocks contain precisely 16 points and there is an embedding $\mathrm{G} \hookrightarrow \mathrm{G}_{6}=G F(2)^{4} \mathrm{wr}_{\mathbf{6}} \mathbf{S}_{\mathbf{6}}$ mapping the inertial group of $\mathrm{G}$ onto the group of translations of $\mathrm{G}_{6}$ corresponding to the $G F(2)$-vector subspace

$$
W=\left\{\left(z_{1}, \ldots, z_{6}\right) \in V=\left(G F(4)^{2}\right)^{6}: \sum_{i=1}^{6} f^{i}\left(z_{i}\right)=0\right\},
$$

where $f$ is the bijective mapping $G F(4)^{2} \rightarrow G F(4)^{2}$ by (12). There are just two nonisomorphic embeddings of $\mathrm{G}$ within $\mathrm{G}_{6}$ which are generated as follows:
1. $\left\langle v \mapsto J_{k}(v)+w: w \in W, k=1, \ldots, 5\right\rangle$
(splits over $\mathrm{N}$ ),
2. $\left\langle v \mapsto J_{k}(v)+u_{k}+w: w \in W, k=1, \ldots, 5\right\rangle$
(does not split over $\mathrm{N}$ ),

where $J_{k}$ are given by (3), (4) and (5) and $u_{k}$ are the vectors (29).

4.5 The case $\overline{\mathrm{G}} \simeq \mathbf{A}_{m+2}$. It remains to examine the case where $\overline{\mathrm{G}}$ is the alternating group $\mathbf{A}_{m+2}$. In such a case $\mathbf{G}$ embeds into $\tilde{\mathrm{G}}=G F(2)^{4} \mathrm{wr}_{\alpha} \mathbf{A}_{m+2}$, where $m=4,5,6$ or 7 and $\alpha$ is the unique representation of $\mathbf{A}_{m+1}$ as a subgroup of $\mathbf{G L}(4,2) \simeq \mathbf{A}_{\mathbf{8}}$. This means that $\tilde{G}$ is one of the four groups $\mathrm{H}_{m+2}$ introduced in 2.4 and 2.5. According to Lemma 3.4.1, for each of the groups $\mathrm{H}_{m+2}$, there is precisely one $\mathbf{A}_{m+2}$-invariant $m$-transversal subspace $W$ such as (13) with functions $f_{i}$ and $g_{i}$ given by (14). Now, we state

4.5.1 Theorem. Let $\overline{\mathrm{G}} \simeq \mathbf{A}_{m+2}$. Then $4 \leq m \leq 7$, the blocks contain precisely 16 points and there is an embedding $\mathrm{G} \hookrightarrow \mathrm{H}_{m+2}=G F(2)^{4} \mathrm{wr} \mathbf{A}_{m+2}$ mapping the inertial group of $\bar{\Omega}$ onto the group $N_{m+2}$ of translations of $\mathrm{H}_{m+2}$ corresponding to the subspace $W_{m+2}$ defined by (13) and (14). The group $\mathrm{G}$ is the splitting extension $\left(G F(2)^{4} \text { wr } \mathbf{A}_{m+2}\right)_{\mid W_{m+2}}$ of $N_{m+2}$ by $\mathbf{A}_{m+2}$.

Proof. It is enough to prove that $G$ splits over $N_{m+2}$. We may regard G as a subgroup of $\mathrm{H}_{9}$ (resp. $\mathrm{H}_{6}$ for $m=4$ ) given as an extension

$$
0_{N_{m+2}} \longrightarrow N_{m+2} \longrightarrow G \stackrel{\lambda}{\longrightarrow} A_{m+2} \longrightarrow 1_{A_{m+2}}
$$

of $N_{m+2}$ by a subgroup $A_{m+2}$ of $\mathrm{H}_{9}$ (resp. $\mathrm{H}_{6}$ ) isomorphic to $\mathbf{A}_{m+2}$. We shall first discuss the case $m=5$.

The alternating group $\mathbf{A}_{\mathbf{7}}$ is generated by the permutations (1375)(26) and (34567). Thus, the matrix $A=Q_{3} Q_{2} Q_{6} Q_{5} Q_{4} Q_{3}$, having the non-zero entries given by the quadratic functions over $G F(4)$ :

$$
\begin{array}{lll}
a_{31}=q^{-2} q_{5} q_{4} q_{3} q_{2}, & a_{62}=q_{6} q_{5} q^{\prime-4}, & a_{73}=q_{5} q_{4} q^{\prime \prime-4}, \\
a_{44}=q^{\prime \prime-2} q_{4} q_{3} q_{2} q^{\prime} q^{\prime \prime}, & a_{15}=q_{2} q^{\prime} q^{\prime \prime} q_{3} q_{2} q^{\prime} q^{\prime \prime} q_{7}, & a_{26}=q^{\prime} q^{\prime \prime} q_{7} q_{2} q^{\prime} q^{\prime \prime} q_{7} q_{6}, \\
a_{57}=q_{7} q_{6} q^{\prime} q^{\prime \prime} q_{7} q_{6} q_{5}, & a_{88}=q_{4} q_{3} q_{7} q_{6} q_{5} q_{4}, & a_{99}=q_{3} q_{2} q_{6} q_{5} q_{4} q_{3},
\end{array}
$$

and the matrix $B=Q_{4} Q_{6}$, the non-zero entries of which are 


$$
\begin{array}{lll}
b_{11}=q_{3} q_{5}, & b_{22}=q_{2} q_{4}, & b_{43}=q^{\prime-1} q_{3}, \\
b_{54}=q^{\prime \prime-1} q_{2}, & b_{65}=q_{7} q^{\prime-1}, & b_{76}=q_{6} q^{\prime \prime-1}, \\
b_{37}=\left(q^{\prime} q^{\prime \prime}\right)^{2} & b_{88}=q_{5} q_{7}, & b_{99}=q_{4} q_{6},
\end{array}
$$

generate a subgroup $\left(\simeq \mathbf{A}_{\mathbf{7}}\right)$ of $\mathrm{H}_{9}$ that we may take as $A_{7}$ in (30). Let

$$
a: x \mapsto A(x)+u_{a}, \quad b: x \mapsto B(x)+u_{b}
$$

be elements in $G$ such that $\lambda(a)=A$ and $\lambda(b)=B$. In view of Lemma 4.3.3,a, we may assume the order of $b$ to be 5 . Moreover $A^{4}=1_{A_{7}}$ yields

$$
w_{a}:=a^{4}(x)+x=u_{a}+A\left(u_{a}\right)+A^{2}\left(u_{a}\right)+A^{3}\left(u_{a}\right) \in W \cap \operatorname{Fix}(A) .
$$

The endomorphism $A^{3}+A^{2}+A+i d_{V}$ maps $W$ into $W \cap \operatorname{Fix}(A) \ni w_{a}$. Also $W$ decomposes into the direct sum of cyclic $K[A]$-submodules $W_{1}, \ldots, W_{5}$ having dimension 4 over $K=G F(2)$ (for instance, the ones generated by

$$
\begin{array}{ll}
\left(f_{4}\left(e_{1}\right), g_{4}\left(e_{1}\right), 0,0,0, e_{1}, 0,0,0\right), & \left(f_{4}\left(e_{2}\right), g_{4}\left(e_{2}\right), 0,0,0, e_{2}, 0,0,0\right), \\
\left(f_{4}\left(e_{3}\right), g_{4}\left(e_{3}\right), 0,0,0, e_{3}, 0,0,0\right), & \left(f_{4}\left(e_{4}\right), g_{4}\left(e_{4}\right), 0,0,0, e_{4}, 0,0,0\right), \\
\left(f_{2}\left(e_{1}\right), g_{2}\left(e_{1}\right), 0, e_{1}, 0,0,0,0,0\right), &
\end{array}
$$

for a fixed basis $e_{1}, e_{2}, e_{3}, e_{4}$ of $\left.G F(2)^{4}\right)$. Thus, if $w \in W \cap \operatorname{Fix}(A)$ and $w_{s}$ is the component of $w$ with respect to $W_{s}(s=1, \ldots, 5)$, then $A\left(w_{s}\right)=w_{s}$. As in $W_{s}$ there is just one non-zero vector fixed by $A$ (the one generating the line $\left.\left(i d_{V}+A+A^{2}+A^{3}\right)\left(W_{s}\right)\right)$, we infer that $W \cap \operatorname{Fix}(A)=\left(i d_{V}+A+A^{2}+A^{3}\right)(W)$. Now, let $\bar{w}_{a} \in W$ solve $\left(i d_{V}+A+A^{2}+A^{3}\right)\left(\bar{w}_{a}\right)=w_{a}$, then, up to a replacement of $u_{a}$ by $u_{a}+\bar{w}_{a}$, we may assume the order of $a$ to be 4 . Now $a^{4}=b^{5}=1_{G}$ imply

$$
u_{a} \in \operatorname{Fix}\left(A+A^{2}+A^{3}\right), \quad u_{b} \in \operatorname{Fix}\left(B+B^{2}+B^{3}+B^{4}\right),
$$

or, equivalently, the coordinates of $u_{a}$ and $u_{b}$ satisfy the equations

$$
\begin{aligned}
& x_{1}+x_{3}+\mathbf{i} x_{3}^{2}+\mathbf{i} y_{3}+\mathbf{i} y_{3}^{2}+x_{5}^{2}+\mathbf{i}^{2} y_{5}+\mathbf{i}^{2} x_{7}^{2}+\mathbf{i} y_{7}=0, \\
& y_{1}+x_{3}+x_{3}^{2}+\mathbf{i}^{2} y_{3}+y_{3}^{2}+\mathbf{i} x_{5}+\mathbf{i} y_{5}+\mathbf{i}^{2} y_{5}^{2}+\mathbf{i} x_{7}+\mathbf{i}^{2} x_{7}^{2}+\mathbf{i} y_{7}+\mathbf{i} y_{7}^{2}=0, \\
& x_{2}+\mathbf{i}^{2} y_{2}+\mathbf{i}^{2} y_{2}^{2}+\mathbf{i}^{2} x_{6}+\mathbf{i} x_{6}^{2}+\mathbf{i} y_{6}=0 \\
& \mathbf{i} x_{4}+\mathbf{i}^{2} x_{4}^{2}+\mathbf{i} y_{4}+\mathbf{i}^{2} y_{4}^{2}=0
\end{aligned}
$$

and

$$
\begin{array}{r}
x_{3}+\mathbf{i}^{2} x_{4}+\mathbf{i} y_{4}+\mathbf{i} y_{4}^{2}+x_{5}+\mathbf{i} x_{5}^{2}+\mathbf{i}^{2} y_{5}+\mathbf{i} y_{5}^{2}+\mathbf{i} x_{6}+\mathbf{i} x_{6}^{2}+\mathbf{i} y_{6}+\mathbf{i}^{2} y_{6}^{2}+\mathbf{i}^{2} x_{7}^{2}=0, \\
y_{3}+x_{4}^{2}+\mathbf{i} y_{4}^{2}+\mathbf{i}^{2} x_{5}+\mathbf{i}^{2} x_{5}^{2}+\mathbf{i}^{2} y_{5}+y_{5}^{2}+x_{6}^{2}+\mathbf{i}^{2} y_{6}+\mathbf{i}^{2} x_{7}+\mathbf{i} x_{7}^{2}+y_{7}=0,
\end{array}
$$

respectively (we are writing vectors as $\left(z_{1}, \ldots, z_{7}, 0,0\right)$ with $\left.z_{i}=\left(x_{i}, y_{i}\right) \in G F(4)^{2}\right)$.

Vectors in $\operatorname{Fix}(B)$ and $\operatorname{Fix}(A B)$ satisfy $\left(i d_{V}+B+B^{2}+B^{3}+B^{4}\right)(x)=x$ and $\left(i d_{V}+A B+(A B)^{2}\right)(x)=x$, respectively. Thus, since $B^{5}=(A B)^{3}=i d_{V}$, we have

$$
\begin{aligned}
& W \cap \operatorname{Fix}(B)=\left(i d_{V}+B+B^{2}+B^{3}+B^{4}\right)(W), \\
& W \cap \operatorname{Fix}(A B)=\left(i d_{V}+A B+(A B)^{2}\right)(W) .
\end{aligned}
$$


Furthermore $W$ decomposes into the direct sum of four cyclic $K[B]$-submodules $W_{1}^{\prime}$, $W_{2}^{\prime}, W_{3}^{\prime}, W_{4}^{\prime}$ of dimension 5 over $K=G F(2)$ (for example, the ones generated by the first four vectors in (31)). As the line $\left(i d_{V}+B+B^{2}+B^{3}+B^{4}\right)\left(W_{j}^{\prime}\right)$ is the subspace of vectors in $W_{j}^{\prime}$ fixed by $B$, we have $\operatorname{dim}(W \cap \operatorname{Fix}(B))=4$. Let

$$
W_{a}:=W \cap \operatorname{Fix}\left(A+A^{2}+A^{3}\right), \quad W_{b}:=W \cap \operatorname{Fix}\left(B+B^{2}+B^{3}+B^{4}\right) .
$$

Then the first equation of (34) gives $\operatorname{dim} W_{b}=16$. Also the vectors

$\left(0,0, \mathbf{i}^{2}, \mathbf{i}, 0,0, \mathbf{i}, 1,0,0,1, \mathbf{i}^{2}, 0,0,0,0,0,0\right),\left(\mathbf{i}^{2}, 1,0,0, \mathbf{i}, 0,0,0,0,1,0,0,1,0,0,0,0,0\right)$, $\left(\mathbf{i}, 1, \mathbf{i}^{2}, \mathbf{i}^{2}, \mathbf{i}^{2}, \mathbf{i}^{2}, 0,0, \mathbf{i}^{2}, 0, \mathbf{i}, 0, \mathbf{i}, 0,0,0,0,0\right),\left(\mathbf{i}^{2}, \mathbf{i}, \mathbf{i}^{2}, \mathbf{i}^{2}, 1,1, \mathbf{i}, 1,1, \mathbf{i}^{2}, \mathbf{i}, 0,0, \mathbf{i}, 0,0,0,0\right)$,

generate a 4-dimensional subspace of $W \cap \operatorname{Fix}(A)$ intersecting $W_{b}$ trivially. Since $\operatorname{dim}_{G F(2)} W=20$ and $W \cap \operatorname{Fix}(A) \subseteq W_{a}$, we have

$$
W=W_{b}+(W \cap \operatorname{Fix}(A))=W_{b}+A^{3}(W \cap \operatorname{Fix}(A))=W_{b}+A^{3}\left(W_{a}\right)=A\left(W_{b}\right)+W_{a} .
$$

Clearly $a b: x \longmapsto A B(x)+A\left(u_{b}\right)+u_{a}$ and $(a b)^{3}$ is a translation in $N_{m+2}$ defined by $\left(i d_{V}+A B+(A B)^{2}\right)\left(A\left(u_{b}\right)+u_{a}\right)$, the order of $A B$ being 3. By (34) and (35) the latter vector $(\in W \cap \operatorname{Fix}(A B))$ can be written as $\left(i d_{V}+A B+(A B)^{2}\right)\left(A\left(w_{b}^{\prime}\right)+w_{a}^{\prime}\right)$ for suitable $w_{a}^{\prime} \in W_{a}$ and $w_{b}^{\prime} \in W_{b}$. Thus, up to a replacement of $u_{a}$ by $u_{a}+w_{a}^{\prime}$ and $u_{b}$ by $u_{b}+w_{b}^{\prime}$, we may assume that the order of $a b$ is 3 , which means

$$
A\left(u_{b}\right)+u_{a} \in \operatorname{Fix}\left(A B+(A B)^{2}\right),
$$

or the coordinates of $A\left(u_{b}\right)+u_{a}$ satisfy the equations

$$
\begin{array}{lr}
x_{1}+\mathbf{i}^{2} x_{3}+\mathbf{i} x_{3}^{2}+\mathbf{i}^{2} y_{3}+\mathbf{i}^{2} y_{3}^{2}+x_{4}+\mathbf{i}^{2} x_{4}^{2}+\mathbf{i}^{2} y_{4}+\mathbf{i} y_{4}^{2}=0, \\
y_{1}+\mathbf{i}^{2} x_{3}^{2}+\mathbf{i}^{2} y_{3}+\mathbf{i} x_{4}^{2}+y_{4}=0, & x_{2}+x_{5}+\mathbf{i}^{2} y_{5}^{2}+x_{6}=0, \\
y_{2}+x_{5}+\mathbf{i}^{2} x_{5}^{2}+\mathbf{i}^{2} y_{5}+\mathbf{i}^{2} y_{5}^{2}+\mathbf{i} x_{6}+x_{6}^{2}+\mathbf{i}^{2} y_{6}^{2}=0, & \mathbf{i}^{2} x_{7}+x_{7}^{2}+\mathbf{i} y_{7}+y_{7}^{2}=0 .
\end{array}
$$

If the order of the transformation $a^{3} b a^{2} b^{2}$ is 2 , then $a$ and $b$ generate a complement of $N_{m+2}$ isomorphic to $\mathbf{A}_{\mathbf{7}}$ (see for instance [4, p. 10]) and our claim is proved. Actually we can always arrange $u_{a}$ in such a way the order of $a^{3} b a^{2} b^{2}$ is 2 by adding to $u_{a}$ a suitable vector $w_{a}^{\prime \prime} \in W$ obtained as follows.

The element $a^{3} b a^{2} b^{2}$ corresponds to the mapping $x \mapsto A^{3} B A^{2} B^{2}(x)+v$, where

$$
v:=\left(A^{3} B A+A^{3} B+A^{2}+A+i d_{V}\right)\left(u_{a}\right)+\left(A^{3} B A^{2} B+A^{3} B A^{2}+A^{3}\right)\left(u_{b}\right) .
$$

The order of $A^{3} B A^{2} B$ being $2,\left(a^{3} b a^{2} b^{2}\right)^{2} \in N_{m+2}$ is the translation of the vector

$$
w:=\left(A^{3} B A^{2} B^{2}+i d_{V}\right)(v) .
$$

The fact that $w \in W$ is a further restriction for $u_{a}$ and $u_{b}$ (in addition to (32) and (36)). Thus it turns out that $w$ has coordinates of the shape

$$
\begin{aligned}
& \left(a, \mathbf{i} a+\mathbf{i}^{2} b, \mathbf{i}(a+b), c+\mathbf{i} d, d+\mathbf{i}(a+b)+\mathbf{i}^{2} c, b+c+d+\mathbf{i} a,\right. \\
& \left.\quad \mathbf{i} c+\mathbf{i}^{2} a, \mathbf{i} d+\mathbf{i}^{2}(a+b+c), \mathbf{i} c, d+\mathbf{i} c, a+b, a+b+\mathbf{i} c+\mathbf{i}^{2} d, \mathbf{i}^{2} d, a+b+d, 0,0,0,0\right) .
\end{aligned}
$$

Now the vector

$\left(a+\mathbf{i} b, b+\mathbf{i}^{2} d, d+\mathbf{i}(a+b+c), c+d+\mathbf{i}^{2}(a+b), b+\mathbf{i} d+\mathbf{i}^{2}(a+c), \mathbf{i}(c+d)+\mathbf{i}^{2}(a+b)\right.$,

$$
\left.a+c+\mathbf{i}^{2} d, a+\mathbf{i} c+\mathbf{i}^{2} d, c+\mathbf{i}^{2} d, a+b+c+d, a+b+d, c, 0,0,0,0,0,0\right),
$$


is in $W \cap \operatorname{Fix}\left(A+A^{2}+A^{3}\right) \cap \operatorname{Fix}\left(A B+(A B)^{2}\right)$ as its coordinates fulfill (14), (33), (37). Also $\left(A^{3} B A^{2} B^{2}+i d_{V}\right)\left(A^{3} B A+A^{3} B+A^{2}+A+i d_{V}\right)$ maps this vector to $w$, so we take it as $w_{a}^{\prime \prime}$ and make $w$ zero, i.e. any extension in $\mathrm{H}_{7}$ of $N_{7}$ by $A_{7}$ splits.

Now let $m \neq 5$. The linear mappings

$$
P_{k}=Q_{2} \ldots Q_{k+1} \quad(k=1, \ldots, m),
$$

with $Q_{l}$ given by (6), with entries $(7)(m=4)$ or $(9)(m>5)$, fulfill (8) for $r=m$; so they generate a translation complement in $\mathrm{H}_{m+2}$, that we can take as $A_{m+2}$ in (30). Then $P_{1}=Q_{2}$ and, for $k>1$, the non-zero entries $p_{r s}^{(k)}$ of the matrix $P_{k}$ are

$$
\begin{aligned}
p_{21}^{(k)} & =q^{-1} q_{2} \ldots q_{k}, & p_{12}^{(k)}=q^{\prime} q^{\prime \prime} q^{-1} q_{2} \ldots q_{k-1}, \\
p_{k+2, k+1}^{(k)} & =q_{t-k} \ldots q_{7} q^{\prime \prime-1}, & p_{k+1, k+2}^{(k)}=q_{t-k+1} \ldots q_{7} q^{\prime \prime-1} q^{\prime} q^{\prime \prime}, \\
p_{r r}^{(k)} & =q_{t-r+2} \ldots q_{t+k-r+1} & (k+2<r), \\
p_{s s}^{(k)} & =q_{t-s+2} \ldots q_{7} q^{\prime \prime-1} q^{\prime} q^{\prime \prime} q^{\prime-1} q_{2} \ldots q_{k-s+1} & (3 \leq s \leq k),
\end{aligned}
$$

where, of course, the indicated products of $q_{l}$ occur just if indices allow. Let

$$
p_{k}: x \mapsto P_{k}(x)+u_{k}
$$

an element in $G$ with $\lambda\left(p_{k}\right)=P_{k}$. We proceed assuming $m>5$.

Up to adding an element in $W_{m+2}$, we may take $u_{k}$ with the eighth and ninth coordinate zero. Thus $p_{1}, \ldots, p_{5}$ generate a subgroup of $\mathrm{H}_{7}$ and we may assume

$$
\begin{aligned}
& p_{1}^{3}=1_{G}, \\
& \left(p_{i+1} p_{i}\right)^{3}=1_{G} \quad(i=1, \ldots, 4), \\
& p_{i}^{2}=1_{G} \\
& (i=2, \ldots, 5), \\
& \left(p_{j} p_{i}\right)^{2}=1_{G} \quad(2 \leq i+1<j \leq 5),
\end{aligned}
$$

every extension in $\mathrm{H}_{7}$ of $N_{7}$ by $\mathbf{A}_{\mathbf{7}}$ being splitting. This means

$$
\begin{array}{rlr}
\text { a) } & \left.u_{1} \in \operatorname{Fix}\left(P_{1}+P_{1}^{2}\right), \quad b_{i}\right) u_{i} \in \operatorname{Fix}\left(P_{i}\right), & (i=2, \ldots, 5), \\
\left.c_{i}\right) & P_{i+1}\left(u_{i}\right)+u_{i+1} \in \operatorname{Fix}\left(P_{i+1} P_{i}+\left(P_{i+1} P_{i}\right)^{2}\right), & (i=1, \ldots, 4), \\
\left.d_{i j}\right) & P_{j}\left(u_{i}\right)+u_{j} \in \operatorname{Fix}\left(P_{j} P_{i}\right), & (2 \leq i+1<j \leq 5) .
\end{array}
$$

As $\operatorname{ker}\left(P_{m \mid W}+i d_{W}\right)=\operatorname{im}\left(P_{m \mid W}+i d_{W}\right)$ and $\left(P_{m} P_{m-1}\right)^{3}=1_{A_{m+2}}$, we may take $p_{m}$ of order 2 with $\left(p_{m} p_{m-1}\right)^{3}=1_{G}$ (use Lemma 4.3.3,a,b). Hence

$$
P_{m}\left(u_{m}\right)=u_{m}, \quad P_{m}\left(u_{m-1}\right)+u_{m} \in \operatorname{Fix}\left(P_{m} P_{m-1}+\left(P_{m} P_{m-1}\right)^{2}\right) .
$$

Now, in order to prove that the extension (30) is splitting we have to provide further replacements of the vectors $u_{k}$ making the transformations $p_{m} p_{k}$ of order 2 for $k=$ $1, \ldots, m-2$. This means to make zero the vectors $v_{k m}=\left(p_{m} p_{k}\right)^{2} \in W_{m+2}$. The vectors $v_{k m}$ are subject to the conditions given by Lemma 4.3.3,c,d, as well as to conditions determined by N. Blackburn in [2, section 1] that may be written as follows:

$$
\begin{array}{rll}
\left.a_{i j}\right) & \left(i d_{V}+P_{j}\right)\left(v_{i m}\right)+\left(i d_{V}+P_{i}\right)\left(v_{j m}\right)=0_{V} & (2<i+1<j<m-1), \\
\left.b_{i}\right) & \left(i d_{V}+P_{1}\right)\left(v_{i m}\right)+\left(P_{i}+P_{m}\right)\left(v_{1 m}\right)=0_{V} & (2<i<m-1), \\
\left.c_{i}\right) & \left(i d_{V}+P_{m} P_{m-1}+\left(P_{m} P_{m-1}\right)^{2}\right)\left(v_{i m}\right)=0_{V} & (i<m-2), \\
d) & \left(i d_{V}+P_{3} P_{2}+\left(P_{3} P_{2}\right)^{2}\right)\left(v_{2 m}+v_{3 m}\right)=0_{V}, & \\
e) & \left(i d_{V}+P_{1} P_{2}+\left(P_{1} P_{2}\right)^{2}\right)\left(P_{1}\left(v_{1 m}\right)+v_{2 m}\right)=0_{V} .
\end{array}
$$


Now we have to discuss separately the cases $m=6$ and $m=7$. Let $m=6$. By Lemma 4.3.3,d, $P_{6} P_{1}$ and $P_{1}+P_{1}^{2}$ leave $v_{16} \in W_{8}$ fixed. This forces $v_{16}$ to have coordinates

$$
\begin{aligned}
& \left(x_{4}+x_{4}^{2}+\mathbf{i} y_{4}+\mathbf{i}^{2} y_{4}^{2}+\mathbf{i} y_{5}+\mathbf{i}^{2} y_{5}^{2}+\mathbf{i} x_{6}+\mathbf{i}^{2} x_{6}^{2}+\mathbf{i} y_{8}+\mathbf{i}^{2} y_{8}^{2},\right. \\
& x_{4}+x_{4}^{2}+\mathbf{i}^{2} y_{4}+\mathbf{i}^{2} y_{5}+\mathbf{i} x_{6}+\mathbf{i}^{2} x_{6}^{2}+y_{6}+\mathbf{i}^{2} y_{6}^{2}+\mathbf{i}^{2} x_{8}+x_{8}^{2}+\mathbf{i} y_{8}+\mathbf{i}^{2} y_{8}^{2}, \\
& \mathbf{i} x_{4}+\mathbf{i}^{2} x_{4}^{2}+\mathbf{i} y_{4}^{2}+y_{5}+\mathbf{i}^{2} y_{5}^{2}+\mathbf{i} y_{6}+y_{6}^{2}+\mathbf{i} x_{8}+y_{8}+y_{8}^{2}, \\
& \mathbf{i} x_{4}^{2}+\mathbf{i} y_{4}+\mathbf{i}^{2} y_{5}+\mathbf{i}^{2} y_{5}^{2}+\mathbf{i} x_{6}+\mathbf{i}^{2} x_{6}^{2}+\mathbf{i}^{2} y_{6}+\mathbf{i} y_{6}^{2}+\mathbf{i}^{2} x_{8}+\mathbf{i} y_{8}+\mathbf{i}^{2} y_{8}^{2}, \\
& \quad \mathbf{i} x_{4}^{2}+y_{4}+\mathbf{i} y_{5}+\mathbf{i} y_{6}+y_{6}^{2}+x_{8}^{2}+y_{8}+y_{8}^{2}, \\
& \quad x_{4}+\mathbf{i} x_{4}^{2}+y_{4}+\mathbf{i}^{2} y_{4}^{2}+\mathbf{i}^{2} y_{5}+\mathbf{i} y_{5}^{2}+x_{6}+\mathbf{i} x_{6}^{2}+y_{6}+\mathbf{i}^{2} y_{6}^{2}+x_{8}+y_{8}+\mathbf{i} y_{8}^{2}, \\
& \left.\quad x_{4}, y_{4}, \mathbf{i}^{2} y_{5}+y_{5}^{2}, y_{5}, x_{6}, y_{6}, \mathbf{i}^{2} x_{8}+\mathbf{i} x_{8}^{2}+\mathbf{i} y_{8}^{2}, x_{8}+\mathbf{i} x_{8}^{2}+\mathbf{i} y_{8}+\mathbf{i}^{2} y_{8}^{2}, x_{8}, y_{8}, 0,0\right)
\end{aligned}
$$

with $x_{41}=y_{42}=x_{61}=y_{62}=0$. Also, by $\left(40, c_{1}\right),\left(P_{6} P_{5}+\left(P_{6} P_{5}\right)^{2}\right)\left(v_{16}\right)=v_{16}$, i.e. $x_{81}=y_{61}$ and $y_{82}=x_{62}$. Consider now, the vector $w_{6} \in W_{8}$ having coordinates

$$
\begin{aligned}
& \left(\mathbf{i} x_{4}^{2}+y_{4}+\mathbf{i} y_{4}^{2}+\mathbf{i} y_{5}^{2}+\mathbf{i} x_{6}^{2}+\mathbf{i}^{2} x_{8}+\mathbf{i}^{2} x_{8}^{2},\right. \\
& x_{4}+\mathbf{i} y_{4}^{2}+\mathbf{i}^{2} y_{5}+\mathbf{i} y_{5}^{2}+\mathbf{i} x_{6}^{2}+y_{6}+\mathbf{i}^{2} y_{6}^{2}+\mathbf{i}^{2} x_{8}+\mathbf{i}^{2} x_{8}^{2}+\mathbf{i} y_{8}+y_{8}^{2}, \\
& \mathbf{i} x_{4}^{2}+\mathbf{i} y_{4}^{2}+y_{5}+\mathbf{i}^{2} y_{5}^{2}+\mathbf{i} x_{6}^{2}+y_{6}+\mathbf{i}^{2} x_{8}+\mathbf{i}^{2} x_{8}^{2}, \\
& \quad \mathbf{i} x_{4}^{2}+\mathbf{i} y_{4}^{2}+\mathbf{i}^{2} y_{5}+\mathbf{i}^{2} y_{5}^{2}+\mathbf{i} y_{6}^{2}+x_{8}+x_{8}^{2}+\mathbf{i}^{2} y_{8}+\mathbf{i} y_{8}^{2}, 0,0, \\
& \quad x_{4}+\mathbf{i} x_{4}^{2}+\mathbf{i} y_{4}^{2}, y_{4}+\mathbf{i} y_{4}^{2}, \mathbf{i} y_{5}+y_{5}^{2}, \mathbf{i}^{2} y_{5}+y_{5}^{2}, \mathbf{i} y_{6}+y_{6}^{2}, x_{6}+\mathbf{i}^{2} y_{6}+\mathbf{i} y_{6}^{2}, \\
& \quad \mathbf{i}^{2} y_{6}+\mathbf{i} y_{6}^{2}+\mathbf{i}^{2} x_{8}+\mathbf{i}^{2} x_{8}^{2}+\mathbf{i}^{2} y_{8}+\mathbf{i} y_{8}^{2}, x_{6}+\mathbf{i}^{2} y_{6}+\mathbf{i} y_{6}^{2}+x_{8}+x_{8}^{2}+y_{8}+\mathbf{i}^{2} y_{8}^{2}, \\
& \left.\quad \mathbf{i} x_{6}^{2}+\mathbf{i}^{2} y_{6}+\mathbf{i} y_{6}^{2}+x_{8}+x_{8}^{2}+y_{8}+\mathbf{i}^{2} y_{8}^{2}, y_{6}+\mathbf{i}^{2} y_{6}^{2}+x_{8}+x_{8}^{2}+\mathbf{i} y_{8}+y_{8}^{2}, 0,0\right) .
\end{aligned}
$$

Looking at $(38)$, we see that $\left(i d_{V}+P_{6} P_{1}\right)\left(w_{6}\right)=v_{16}$; thus we can make $p_{6} p_{1}$ of order 2 by replacing $u_{6}$ by $u_{6}+w_{6}$. Notice that $P_{6}$ and $P_{6} P_{5}+\left(P_{6} P_{5}\right)^{2}$ leave $w_{6}$ fixed and this guarantees that the conditions $p_{6}^{2}=1_{G}$ and $\left(p_{6} p_{5}\right)^{3}=1_{G}$ still hold.

As $v_{26}$ is contained in $W_{8} \cap \operatorname{Fix}\left(P_{2}\right) \cap \operatorname{Fix}\left(P_{6}\right)$ by Lemma 4.3.3,c, $v_{26}$ has cordinates $\left(\mathbf{i}^{2} x_{4}+x_{4}^{2}+x_{5}+\mathbf{i}^{2} x_{5}^{2}+\mathbf{i}^{2} y_{8}+y_{8}^{2}, \mathbf{i} x_{4}^{2}+\mathbf{i}^{2} x_{5}^{2}+y_{6}^{2}+y_{8}, \mathbf{i}^{2} x_{4}+x_{4}^{2}+x_{5}+\mathbf{i}^{2} x_{5}^{2}+\mathbf{i}^{2} y_{8}+y_{8}^{2}\right.$,

$\mathbf{i}^{2} x_{4}+\mathbf{i} x_{4}^{2}+\mathbf{i} y_{6}^{2}+y_{8}+y_{8}^{2}, \mathbf{i} x_{4}, x_{4}+\mathbf{i} x_{4}^{2}, x_{4}, x_{4}+\mathbf{i} x_{4}^{2}$,

$\left.x_{5}, \mathbf{i}^{2} x_{5}, 0, y_{6}, \mathbf{i}^{2} y_{8}^{2}, \mathbf{i}^{2} y_{8}, \mathbf{i} y_{8}+\mathbf{i} y_{8}^{2}, y_{8}, 0,0\right)$,

where $x_{51}=x_{52}$ and $y_{61}=0$. Also $\left(40, c_{2}, e\right)$ give $y_{81}=0$ and $x_{51}=y_{62}=y_{82}$, so

$$
\begin{aligned}
v_{26}= & \left(\left(\mathbf{i}\left(a_{26}+b_{26}\right), b_{26}+\mathbf{i} a_{26}, \mathbf{i}\left(a_{26}+b_{26}\right), a_{26}, \mathbf{i} a_{26}+\mathbf{i}^{2} b_{26},\right.\right. \\
& \left.\mathbf{i}^{2}\left(a_{26}+b_{26}\right), a_{26}+\mathbf{i} b_{26}, \mathbf{i}^{2}\left(a_{26}+b_{26}\right), \mathbf{i}^{2} c_{26}, \mathbf{i} c_{26}, 0, \mathbf{i} c_{26}, \mathbf{i} c_{26}, c_{26}, \mathbf{i} c_{26}, \mathbf{i} c_{26}, 0,0\right) .
\end{aligned}
$$

Now the vectors

$$
\begin{aligned}
& w_{2}=\left(0,0,0,0,0,0,0,0, \mathbf{i} c_{26}, c_{26}, \mathbf{i} c_{26}, \mathbf{i} c_{26}, \mathbf{i} c_{26}, c_{26}, 0,0,0,0\right), \\
& w_{6}=\left(b_{26}, \mathbf{i} a_{26}+\mathbf{i}^{2} b_{26}, a_{26}, \mathbf{i}\left(a_{26}+b_{26}\right), \mathbf{i} a_{26}+\mathbf{i}^{2} b_{26}, \mathbf{i}^{2}\left(a_{26}+b_{26}\right), 0, \ldots, 0\right),
\end{aligned}
$$

fulfill $\left(i d_{V}+P_{6}\right)\left(w_{2}\right)+\left(i d_{V}+P_{2}\right)\left(w_{6}\right)=v_{26}$ and a replacement of $u_{2}$ by $u_{2}+w_{2}$ and $u_{6}$ by $u_{6}+w_{6}$ yields $\left(p_{6} p_{2}\right)^{2}=1_{G}$. Also $w_{2} \in \operatorname{Fix}\left(P_{2}\right) \cap \operatorname{Fix}\left(P_{2} P_{1}+\left(P_{2} P_{1}\right)^{2}\right)$, whereas $w_{6} \in \operatorname{Fix}\left(P_{6}\right) \cap \operatorname{Fix}\left(P_{6} P_{5}+\left(P_{6} P_{5}\right)^{2}\right) \cap \operatorname{Fix}\left(P_{6} P_{1}\right)$, or the conditions $p_{2}^{2}=\left(p_{2} p_{1}\right)^{3}=p_{6}^{2}=$ $\left(p_{6} p_{5}\right)^{3}=\left(p_{6} p_{1}\right)^{2}=1_{G}$ are preserved. Unfortunately $P_{3}\left(w_{2}\right) \notin \operatorname{Fix}\left(P_{3} P_{2}+\left(P_{3} P_{2}\right)^{2}\right)$, thus we have to restore $\left(39, c_{2}\right)$. This can be done by replacing $u_{3}$ by $u_{3}+w_{3}$ with 
$w_{3}=\left(\mathbf{i} c_{26}, c_{26}, \mathbf{i} c_{26}, \mathbf{i} c_{26}, \mathbf{i} c_{26}, c_{26}, 0,0,0,0,0,0,0,0,0,0,0,0\right)$.

As we made $v_{16}$ zero, $\left(40, b_{3}\right)$ forces $v_{36}$ to be stable under $P_{1}$. Also, since $v_{26}=0_{V}$, condition $(40, d)$ yields $v_{36} \in \operatorname{Fix}\left(P_{3} P_{2}+\left(P_{3} P_{2}\right)^{2}\right)$, and this occurs just if $v_{36}=0$. Likewise $v_{46} \in \operatorname{Fix}\left(P_{1}\right)$ by $\left(40, b_{4}\right)$, hence

$$
\begin{aligned}
v_{26}= & \left(\mathbf{i}^{2} x_{3}+\mathbf{i} x_{3}^{2}+\mathbf{i}^{2} y_{3}+y_{3}^{2}, \mathbf{i} x_{3}+\mathbf{i}^{2} x_{3}^{2}+\mathbf{i}^{2} y_{3}+\mathbf{i}^{2} y_{3}^{2}, \mathbf{i}^{2} x_{3}+\mathbf{i} x_{3}^{2}+\mathbf{i} y_{3},\right. \\
& \left.\mathbf{i}^{2} x_{3}+\mathbf{i}^{2} x_{3}^{2}+\mathbf{i}^{2} y_{3}+\mathbf{i} y_{3}^{2}, x_{3}, y_{3}, 0, \ldots, 0\right) .
\end{aligned}
$$

Also $v_{46} \in \operatorname{Fix}\left(P_{6}\right)$ by Lemma $4.3 .3, c$, so $y_{3}=\mathbf{i}^{2} x_{3}+\mathbf{i}^{2} x_{3}^{2}$. Besides by $\left(40, a_{24}\right)$ it follows $P_{2}\left(v_{46}\right)=v_{46}$, which means $x_{3}=0$ and then $v_{46}=0$. Thus $p_{1}, \ldots, p_{6}$ generate a translation complement in $\mathrm{H}_{8}$ isomorphic to $\mathbf{A}_{\mathbf{8}}$, i.e. the extension (30) splits.

Let $m=7$. Thanks to Lemma $4.3 .3, d$, the vector $v_{17}$ has coordinates

$$
\begin{aligned}
& \left(x_{4}+\mathbf{i} x_{5}^{2}+\mathbf{i}^{2} x_{6}+\mathbf{i}^{2} x_{6}^{2}+y_{6}+y_{6}^{2}+y_{7}+\mathbf{i} x_{9}+x_{9}^{2}+y_{9}+\mathbf{i}^{2} y_{9}^{2},\right. \\
& \mathbf{i} x_{4}^{2}+x_{5}+\mathbf{i}^{2} x_{6}+\mathbf{i}^{2} x_{6}^{2}+\mathbf{i} y_{6}+\mathbf{i} y_{6}^{2}+\mathbf{i} y_{7}^{2}+\mathbf{i} x_{9}+x_{9}^{2}+\mathbf{i}^{2} y_{9}+\mathbf{i} y_{9}^{2}, \\
& x_{4}+\mathbf{i}^{2} x_{5}+\mathbf{i}^{2} x_{5}^{2}+\mathbf{i} x_{6}+\mathbf{i} y_{6}^{2}+\mathbf{i}^{2} y_{7}^{2}+\mathbf{i} x_{9}+x_{9}^{2}+\mathbf{i} y_{9}+\mathbf{i} y_{9}^{2}, \\
& \quad \mathbf{i} x_{5}+\mathbf{i}^{2} x_{6}+\mathbf{i} y_{6}^{2}+\mathbf{i} y_{7}^{2}+\mathbf{i} x_{9}+\mathbf{i}^{2} x_{9}^{2}+y_{9}+\mathbf{i} y_{9}^{2}, \\
& \mathbf{i}^{2} x_{4}+\mathbf{i}^{2} x_{4}^{2}+x_{5}^{2}+\mathbf{i}^{2} x_{6}^{2}+\mathbf{i} y_{6}^{2}+\mathbf{i}^{2} y_{7}+y_{7}^{2}+\mathbf{i} x_{9}^{2}+\mathbf{i}^{2} y_{9}+\mathbf{i}^{2} y_{9}^{2}, \\
& \quad x_{4}+\mathbf{i} x_{4}^{2}+\mathbf{i} x_{5}^{2}+\mathbf{i} x_{6}+\mathbf{i}^{2} y_{6}+\mathbf{i} y_{6}^{2}+y_{7}+\mathbf{i}^{2} y_{7}^{2}+x_{9}+\mathbf{i} x_{9}^{2}+y_{9}, x_{4}, \mathbf{i}^{2} x_{4}+\mathbf{i}^{2} x_{4}^{2}, x_{5}, \\
& \left.\quad x_{5}+\mathbf{i} x_{5}^{2}, x_{6}, y_{6}, \mathbf{i} y_{7}+y_{7}^{2}, y_{7}, \mathbf{i}^{2} x_{9}+\mathbf{i}^{2} x_{9}^{2}+\mathbf{i}^{2} y_{9}, \mathbf{i}^{2} x_{9}+x_{9}^{2}+y_{9}+\mathbf{i} y_{9}^{2}, x_{9}, y_{9}\right) .
\end{aligned}
$$

with $x_{61}=x_{62}$ and $y_{61}=0$. Besides, by $\left(40, c_{1}\right),\left(P_{7} P_{6}+\left(P_{7} P_{6}\right)^{2}\right)\left(v_{17}\right)=v_{17}$, which means $y_{91}=y_{71}+y_{72}+x_{91}+x_{92}$ and $y_{92}=y_{71}+x_{92}$. Let

$$
\begin{gathered}
w_{7}:=\left(x_{4}+x_{4}^{2}+x_{5}+\mathbf{i} x_{5}^{2}+\mathbf{i} x_{6}+x_{6}^{2}+y_{6}+y_{6}^{2}+\mathbf{i}^{2} y_{7}^{2}+x_{9}+\mathbf{i}^{2} x_{9}^{2},\right. \\
x_{4}+\mathbf{i} x_{4}^{2}+x_{5}+\mathbf{i}^{2} x_{5}^{2}+\mathbf{i}^{2} x_{6}+\mathbf{i} x_{6}^{2}+y_{6}+y_{6}^{2}+\mathbf{i} y_{7}+\mathbf{i}^{2} y_{7}^{2}+\mathbf{i} x_{9}^{2}, \\
x_{4}+\mathbf{i}^{2} x_{5}+\mathbf{i}^{2} x_{5}^{2}+\mathbf{i}^{2} x_{6}+\mathbf{i} x_{6}^{2}+y_{6}+y_{6}^{2}+y_{7}+\mathbf{i}^{2} x_{9}^{2}, \\
\mathbf{i} x_{5}+x_{6}+\mathbf{i}^{2} x_{6}^{2}+y_{6}+y_{6}^{2}+\mathbf{i}^{2} y_{7}+y_{7}^{2}+\mathbf{i} x_{9}^{2}, 0,0, \mathbf{i}^{2} x_{4}+x_{4}^{2}, \\
\mathbf{i} x_{4}+\mathbf{i} x_{4}^{2}, \mathbf{i} x_{5}^{2}, x_{5}, \mathbf{i} y_{6}+\mathbf{i} y_{6}^{2}, \mathbf{i}^{2} x_{6}+\mathbf{i} x_{6}^{2}+\mathbf{i} y_{6}+\mathbf{i} y_{6}^{2}, \mathbf{i}^{2} y_{7}, \mathbf{i} y_{7}, \\
\left.y_{7}^{2}+x_{9}+\mathbf{i}^{2} x_{9}^{2}, \mathbf{i}^{2} y_{7}+\mathbf{i} y_{7}^{2}+\mathbf{i} x_{9}+\mathbf{i} x_{9}^{2}, y_{7}^{2}+x_{9}^{2}, \mathbf{i} y_{7}+\mathbf{i}^{2} y_{7}^{2}+\mathbf{i} x_{9}\right) .
\end{gathered}
$$

We have $w_{7} \in W_{9}$ and $\left(i d_{V}+P_{7} P_{1}\right)\left(w_{7}\right)=v_{17}$. Thus we can make $p_{7} p_{1}$ of order 2 by replacing $u_{7}$ by $u_{7}+w_{7}$. Notice that $w_{7}$ is fixed by both $P_{7}$ and $P_{7} P_{6}+\left(P_{7} P_{6}\right)^{2}$, so that the conditions $p_{7}^{2}=1_{G}$ and $\left(p_{7} p_{6}\right)^{3}=1_{G}$ are both preserved.

Consider now the vector $v_{27} \in W_{9} \cap \operatorname{Fix}\left(P_{2}\right) \cap \operatorname{Fix}\left(P_{7}\right)$ having coordinates

$$
\begin{aligned}
& \left(\mathbf{i}^{2} x_{4}+\mathbf{i}^{2} y_{4}+y_{4}^{2}+\mathbf{i} x_{5}+\mathbf{i} y_{6}+\mathbf{i}^{2} y_{6}^{2}+\mathbf{i} y_{7}+\mathbf{i} y_{9}^{2}, \mathbf{i} x_{4}^{2}+y_{4}+\mathbf{i} y_{4}^{2}+\mathbf{i}^{2} x_{5}^{2}+\mathbf{i} y_{6}+\mathbf{i}^{2} y_{6}^{2}+\mathbf{i}^{2} y_{7}^{2}+\mathbf{i}^{2} y_{9},\right. \\
& x_{4}+x_{4}^{2}+\mathbf{i} y_{4}+\mathbf{i}^{2} y_{4}^{2}+\mathbf{i} y_{6}+\mathbf{i}^{2} y_{6}^{2}+\mathbf{i}^{2} y_{7}+\mathbf{i} y_{7}^{2}+\mathbf{i} y_{9}+\mathbf{i}^{2} y_{9}^{2}, \\
& \quad \mathbf{i} x_{4}^{2}+y_{4}+\mathbf{i} y_{4}^{2}+\mathbf{i}^{2} x_{5}^{2}+\mathbf{i} y_{6}+\mathbf{i}^{2} y_{6}^{2}+\mathbf{i}^{2} y_{7}^{2}+\mathbf{i}^{2} y_{9}, x_{4}+x_{4}^{2}+y_{4}^{2}, \\
& \left.\quad x_{4}+\mathbf{i} x_{4}^{2}+\mathbf{i}^{2} y_{4}+y_{4}^{2}, x_{4}, y_{4}, x_{5}, \mathbf{i}^{2} x_{5}, \mathbf{i} y_{6}+\mathbf{i} y_{6}^{2}, y_{6}, \mathbf{i} y_{7}, y_{7}, y_{9}+\mathbf{i}^{2} y_{9}^{2}, \mathbf{i} y_{9}^{2}, \mathbf{i} y_{9}, y_{9}\right),
\end{aligned}
$$

with $x_{41}=y_{41}=x_{51}=y_{61}=y_{72}=0$. By $\left(40, c_{2}, e\right) v_{27}$ is also fixed by $P_{7} P_{6}$ and $P_{1} P_{2}+\left(P_{1} P_{2}\right)^{2}$, so it has coordinates

$v_{27}=\left(a_{27}+\mathbf{i} b_{27}, a_{27}+\mathbf{i}^{2} b_{27}, a_{27}+b_{27}, a_{27}+\mathbf{i}^{2} b_{27}, a_{27}+\mathbf{i}^{2} b_{27}, \mathbf{i} b_{27}+\mathbf{i}^{2} a_{27}, \mathbf{i} a_{27}, \mathbf{i} b_{27}\right.$, $\left.\mathbf{i} c_{27}, c_{27}, \mathbf{i} d_{27}, \mathbf{i} d_{27}, \mathbf{i}\left(c_{27}+d_{27}\right), c_{27}+d_{27}, \mathbf{i} d_{27}, c_{27}+\mathbf{i}^{2} d_{27}, d_{27}+\mathbf{i}^{2} c_{27}, \mathbf{i} c_{27}+\mathbf{i}^{2} d_{27}\right)$. 
Now, in view of Lemma $4.3 .3, c$ and $\left(40, b_{i}\right)$, we can write the vectors $v_{i 7}, i>2$, as:

$$
\begin{aligned}
& v_{37}=\left(\mathbf{i} a_{37}, a_{37}, \mathbf{i} a_{37}, \mathbf{i} a_{37}, \mathbf{i} a_{37}, a_{37}, 0, \ldots, 0\right), \\
& v_{47}=\left(\mathbf{i}^{2} a_{47}, \mathbf{i}^{2} a_{47}, \mathbf{i} a_{47}, \mathbf{i}^{2} a_{47}, \mathbf{i}^{2} a_{47}, \mathbf{i} a_{47}, 0, \ldots, 0\right), \\
& v_{57}=\left(\mathbf{i} a_{57}, a_{57}, \mathbf{i} a_{57}, \mathbf{i} a_{57}, \mathbf{i} a_{57}, a_{57}, 0, \ldots, 0\right) .
\end{aligned}
$$

These vectors are related to $v_{27}$ via $\left(40, a_{2 i}\right)$ and $(40, d)$; in particular we have

$$
c_{27}=d_{27}=0, \quad a_{37}=a_{27}+b_{27}, \quad a_{47}=a_{27}, \quad a_{57}=a_{27}+b_{27} .
$$

Now $w_{7}=\left(a_{27}+\mathbf{i}^{2} b_{27}, \mathbf{i} a_{27}+\mathbf{i}^{2} b_{27}, \mathbf{i} b_{27}, a_{27}+\mathbf{i}^{2} b_{27}, a_{27}+\mathbf{i}^{2} b_{27}, \mathbf{i} b_{27}+\mathbf{i}^{2} a_{27}, 0, \ldots, 0\right)$ fulfills $\left(i d_{V}+P_{2}\right)\left(w_{7}\right)=v_{27}$, so the replacement of $u_{7}$ by $u_{7}+w_{7}$ makes $p_{7} p_{2}$ of order 2. Notice that $P_{7}\left(w_{7}\right)=P_{7} P_{1}\left(w_{7}\right)=\left(P_{7} P_{6}+\left(P_{7} P_{6}\right)^{2}\right)\left(w_{7}\right)=w_{7}$ and this ensures that the already achieved conditions on $p_{i}$ are not destroyed. By (41) $v_{27}=0$ yields now $v_{i 7}=0$ for all $i>2$ and we conclude that the extension (30) splits.

Finally, let $m=4$. By Lemma 4.3.3, $a, b$, we may take $p_{1}$ of order 3 and, for $l>1, p_{l}$ of order 2 with $\left(p_{l} p_{l-1}\right)^{3}=1_{G}, u_{l} \in \operatorname{Fix}\left(P_{l}\right)$ and $P_{l}\left(u_{l-1}\right)+u_{l} \in \operatorname{Fix}\left(P_{l} P_{l-1}+\left(P_{l} P_{l-1}\right)^{2}\right)$. Thus we have only to transform to zero the vectors $v_{i j}=\left(p_{j} p_{i}\right)^{2} \in W_{6}$ with $|j-i|>1$, being careful to retain the achieved conditions. We have to proceed analogously to the case $m>5$. By Lemma 4.3.3,c, $d$, it turns out that $v_{24}$ is fixed by $P_{2}$ and $P_{4}$, whereas $\left(P_{i} P_{1}\right)\left(v_{1 i}\right)=\left(P_{1}+P_{1}^{2}\right)\left(v_{1 i}\right)=v_{1 i}(i=3,4)$. Thus we can represent the vectors $v_{i j}$ as

$$
\begin{gathered}
v_{13}=\left(\mathbf{i}^{2} a_{13}^{2}+\mathbf{i} b_{13}^{2}+\mathbf{i} c_{13}^{2}, 0, \mathbf{i}^{2} a_{13}+b_{13}+c_{13}, \mathbf{i} a_{13}+b_{13}+\mathbf{i} c_{13}, \mathbf{i} a_{13}^{2}+\mathbf{i}^{2} b_{13}^{2}+\mathbf{i} c_{13}^{2},\right. \\
\left.a_{13}^{2}+b_{13}^{2}, a_{13}, b_{13}, \mathbf{i} a_{13}^{2}, \mathbf{i} a_{13}^{2}+\mathbf{i}^{2} b_{13}^{2}, c_{13}, \mathbf{i} c_{13}\right), \\
v_{14}=\left(\mathbf{i} a_{14}^{2}+\mathbf{i} b_{14}^{2}+c_{14}, \mathbf{i} a_{14}^{2}+\mathbf{i} b_{14}^{2}+c_{14}, a_{14}, a_{14}+\mathbf{i} b_{14}+\mathbf{i}^{2} c_{14}^{2}, \mathbf{i} a_{14}^{2}+b_{14}^{2}+\mathbf{i}^{2} c_{14},\right. \\
\mathbf{i} a_{14}^{2}+\mathbf{i}^{2} b_{14}^{2}+\mathbf{i} c_{14}, b_{14}, b_{14}, c_{14}, \mathbf{i} a_{14}^{2}+\mathbf{i}^{2} b_{14}^{2}+c_{14}, a_{14}+\mathbf{i}^{2} b_{14}+\mathbf{i} c_{14}^{2}, \\
\left.\mathbf{i}^{2} a_{14}+\mathbf{i} b_{14}+\mathbf{i} c_{14}^{2}\right), \\
v_{24}=\left(a_{24}+b_{24}, \mathbf{i}\left(a_{24}+b_{24}\right), \mathbf{i}\left(a_{24}+b_{24}\right)^{2}, 0, a_{24}, \mathbf{i} a_{24}, \mathbf{i} a_{24}^{2}, 0, b_{24}, \mathbf{i} b_{24}, \mathbf{i} b_{24}^{2}, 0\right) .
\end{gathered}
$$

Furthermore, it follows from [2, section 1] that the vectors $v_{i j}$ fulfill the conditions

$$
\begin{aligned}
& \text { a) }\left(i d_{V}+P_{4} P_{3}+\left(P_{4} P_{3}\right)^{2}\right)\left(v_{13}+v_{14}\right)=0_{V} \\
& \text { b) }\left(i d_{V}+P_{1} P_{2}+\left(P_{1} P_{2}\right)^{2}\right)\left(P_{1}\left(v_{14}\right)+v_{24}\right)=0_{V}
\end{aligned}
$$

We can annihilate each of $v_{i j}$ successively. Adding

$w_{4}=\left(\mathbf{i}^{2} a_{24}+\mathbf{i} b_{24}, \mathbf{i}^{2} a_{24}+b_{24}, \mathbf{i}^{2} a_{24}^{2}+b_{24}^{2}, \mathbf{i} a_{24}^{2}+\mathbf{i} b_{24}^{2}, a_{24}+b_{24}, \mathbf{i} a_{24}+\mathbf{i} b_{24}, \mathbf{i} b_{24}^{2}, 0, b_{24}, 0,0, \mathbf{i} b_{24}^{2}\right)$ to $u_{4}$ we make $v_{24}=0$ (in fact, $\left.\left(i d_{V}+P_{2}\right)\left(w_{4}\right)=v_{24}\right)$ and this implies, with the aid of $(42, b), c_{14}=\mathbf{i}^{2} a_{14}^{2}+b_{14}^{2}$. Adding the vector $w_{1}$ with coordinates

$\left(\mathbf{i} a_{14}^{2}+b_{14}^{2}, \mathbf{i}^{2} b_{14}^{2}, b_{14}, \mathbf{i}^{2} a_{14}+\mathbf{i} b_{14}, \mathbf{i}^{2} a_{14}^{2}+\mathbf{i}^{2} b_{14}^{2}, \mathbf{i}^{2} a_{14}^{2}+\mathbf{i} b_{14}^{2}, b_{14}, 0, a_{14}^{2}+\mathbf{i} b_{14}^{2}, \mathbf{i} a_{14}^{2}+\mathbf{i}^{2} b_{14}^{2}, 0,0\right)$ 
to $u_{1}$ we make $v_{14}=0$, (in fact, $\left.\left(i d_{V}+P_{4} P_{1}\right)\left(P_{4}\left(w_{1}\right)\right)=v_{14}\right)$, which implies, together $(42, a), c_{13}=\mathbf{i} a_{13}+\mathbf{i} b_{13}$. Now we can add the vector

$$
\begin{gathered}
w_{1}=\left(\mathbf{i}^{2} b_{13}^{2}, \mathbf{i} a_{13}^{2}, a_{13}+\mathbf{i}^{2} b_{13}, \mathbf{i}^{2} a_{13}+\mathbf{i} b_{13}, \mathbf{i} a_{13}^{2}+\mathbf{i} b_{13}^{2}, \mathbf{i} a_{13}^{2}+b_{13}^{2}, 0,\right. \\
\left.\mathbf{i} a_{13}+\mathbf{i}^{2} b_{13}, a_{13}^{2}+b_{13}^{2}, \mathbf{i} a_{13}^{2}+\mathbf{i} b_{13}^{2}, \mathbf{i}^{2} a_{13}+\mathbf{i}^{2} b_{13}, 0\right)
\end{gathered}
$$

to $u_{1}$ in order to get $v_{13}=0$ (in fact, $\left.\left(i d_{V}+P_{3} P_{1}\right)\left(P_{3}\left(w_{1}\right)\right)=v_{13}\right)$. Notice that all of the added vectors $w_{k}$ do not violate the previous achieved conditions. Thus we can conclude that the extension (30) is in any case splitting.

\section{References}

[1] W. Benz, Vorlesungen über Geometrie der Algebren, Springer-Verlag, Berlin Heidelberg New York 1973.

[2] N. Blackburn, The extension theory of the symmetric and alternating groups, Math. Z. 117 (1970), $191-206$.

[3] P.J. Cameron, Permutation groups, in Handbook of Combinatorics, Vol. 1, Eds. R.L. Graham, M. Grötschel and L. Lovász, Elsevier Science B.V., Amsterdam, MIT Press, Cambridge, MA, 1995.

[4] J.H. Conway, R.T. Curtis, S.P. Norton, R.A. Parker, R.A. Wilson, Atlas of finite groups, Clarendon Press Oxford 1985;

[5] L.E. Dickson, Linear groups, Teubner, Leipzig 1901;

[6] M. Hall, On a theorem of Jordan, Pacific J. Math. 4 (1954), 219 - 226.

[7] C. Hering, Transitive linear groups and linear groups which contain irreducible subgroups of prime order, Geom. Ded. 2 (1974), 425 - 460.

[8] B. Huppert, Endliche Gruppen I, Springer-Verlag, Berlin Heidelberg New York 1967.

[9] W.M. Kantor, Homogeneous designs and geometric lattices, J. Combin. Theory Ser. A 38 no. 1 (1985), $66-74$.

[10] H. Kurzweil, B. Stellmacher, Theorie der endlichen Gruppen, Springer-Verlag, Berlin, Heidelberg, New York 1998.

[11] M.W. Liebeck, The affine permutation groups of rank three, Proc. London Math. Soc. (3) 54 no. 3 (1987), $477-516$.

Dip. Matematica e applicaz. Universitá di Palermo,

Via Archirafi 34,

I-90123 Palermo, Italy

cg@math.unipa.it
Authors' address:

Mathematische Institut Universität Erlangen-Nürnberg

Bismarchstr. $1 \frac{1}{2}$

D-91054 Erlangen, Germany

musumeci@math.unipa.it_strambach@mi.uni-erlangen.de 